ESAIM: M2AN 50 (2016) 965-993

DOI: $10.1051 / \mathrm{m} 2 \mathrm{an} / 2015061$
ESAIM: Mathematical Modelling and Numerical Analysis

www.esaim-m2an.org

\title{
STABILITY ANALYSIS AND ERROR ESTIMATES OF AN EXACTLY DIVERGENCE-FREE METHOD FOR THE MAGNETIC INDUCTION EQUATIONS *
}

\author{
HE YANG ${ }^{1}$ AND FENGYAN LI ${ }^{2}$
}

\begin{abstract}
In this paper, we consider an exactly divergence-free scheme to solve the magnetic induction equations. This problem is motivated by the numerical simulations of ideal magnetohydrodynamic (MHD) equations, a nonlinear hyperbolic system with a divergence-free condition on the magnetic field. Computational methods without satisfying such condition may lead to numerical instability. One class of methods, constrained transport schemes, is widely used as divergence-free treatments. So far there is not much analysis available for such schemes. In this work, we take an exactly divergencefree scheme proposed by [ $\mathrm{Li}$ and Xu, J. Comput. Phys. 231 (2012) 2655-2675] as a candidate of the constrained transport schemes, and adapt it to solve the magnetic induction equations. For the resulting scheme applied to the equations with a constant velocity field, we carry out von Neumann analysis for numerical stability on uniform meshes. We also establish the stability and error estimates based on energy methods. In particular, we identify the stability mechanism due to the spatial and temporal discretizations, and the role of the exactly divergence-free property of the numerical solution for stability. The analysis based on energy methods can be extended to non-uniform meshes, and they can also be applied to the magnetic induction equations with a variable velocity field, which is more relevant to the MHD simulations.
\end{abstract}

Mathematics Subject Classification. 65M60, 65M06, 65M15, 65M12, 35Q85, 35L50.

Received March 23, 2015. Revised July 7, 2015.

\section{INTRODUCTION}

The focus of this paper is to analyze an exactly divergence-free method for magnetic induction equations. This system is extracted from ideal magnetohydrodynamic (MHD) equations, which describe the phenomena of electrically conducting fluids and can model many problems in astrophysics and engineering. The ideal MHD equations consist of a set of nonlinear hyperbolic equations with a divergence-free constraint on the magnetic field, i.e. $\nabla \cdot \mathbf{B}=0$. If such constraint is satisfied initially with compatible boundary conditions, the exact

Keywords and phrases. Stability, error estimates, ideal magnetohydrodynamic (MHD) equations, constrained transport, divergence-free, discontinuous galerkin, magnetic induction equations.

* This research is partially supported by NSF CAREER award DMS-0847241 and NSF DMS-1318409.

1 Department of Radiology, Stanford University, Stanford, CA 94305-5105, USA. hyang2@stanford.edu.

Present address: Department of Mathematics, Ohio State University, Columbus, OH 43210-1174, USA. yang.1671@osu.edu

2 Department of Mathematical Sciences, Rensselaer Polytechnic Institute, Troy, NY 12180-3590, USA. lif@rpi.edu 
solution will satisfy this condition for all time. Numerical methods which violate the condition may lead to nonphysical solutions or numerical instability for some problems [3, 5, 11,22].

One widely used family of approaches to numerically handle the divergence-free condition is called constrained transport (CT), which was introduced by Evans and Hawley in [8]. The basic idea of a CT scheme, at least in its original formulation, is to work with the integral form of the magnetic induction equations to maintain the divergence-free condition exactly or in some discrete sense. The method can also be viewed as a predictorcorrector approach to approximate the magnetic field. The CT framework was further combined with many discretizations, such as Godunov (upwind) or central type finite volume, finite difference, finite element methods with second or higher order accuracy $[2,10,14,20,22]$. Recently there have also been developments on devising CT type methods with the computed magnetic field to be exactly divergence-free $[1,12,14,21]$. The commonly available analysis in literature for CT methods is to show the computed magnetic field is (exactly) divergencefree at time $t=t^{n+1}$ as long as it is at the previous time $t=t^{n}$. In this work, we want to gain further theoretical understanding towards CT type methods.

Given that the ideal MHD equations are nonlinear and hyperbolic, we here take the magnetic induction equations, the equations satisfied by the magnetic field in the ideal MHD system, as a model problem, and adapt to it an exactly divergence-free method based on the methods developed in [13] for ideal MHD equations. Using the resulting scheme as a candidate, we carry out the analysis in order to understand some stability mechanisms of CT methods, the role of the divergence-free property of the computed magnetic field, and the accuracy of the methods.

In this work, we specifically consider the two-dimensional magnetic induction equations,

$$
\left\{\begin{array}{l}
\partial_{t} \mathbf{B}+\widehat{\nabla} \times E_{z}(\mathbf{u}, \mathbf{B})=0, \\
\nabla \cdot \mathbf{B}=0,
\end{array}\right.
$$

where $\mathbf{B}=\left[B_{x}, B_{y}\right]^{\top}$ is the magnetic field, $\mathbf{u}=\left[u_{x}, u_{y}\right]^{\top}$ is a given velocity field, and $E_{z}(\mathbf{u}, \mathbf{B})=u_{y} B_{x}-u_{x} B_{y}$ is the $z$-component of the electric field $\mathbf{E}=-\mathbf{u} \times \mathbf{B}$. In addition, $\widehat{\nabla} \times E_{z}:=\left[\frac{\partial E_{z}}{\partial y},-\frac{\partial E_{z}}{\partial x}\right]^{\top}$, and it corresponds to the first two components of $\nabla \times\left[0,0, E_{z}\right]^{\top}$. In two dimensions, (1.1) can be rewritten as

$$
\left\{\begin{array}{l}
\frac{\partial B_{x}}{\partial t}+\frac{\partial E_{z}}{\partial y}=0 \\
\frac{\partial B_{y}}{\partial t}-\frac{\partial E_{z}}{\partial x}=0 \\
\frac{\partial B_{x}}{\partial x}+\frac{\partial B_{y}}{\partial y}=0
\end{array}\right.
$$

The exactly divergence-free methods in [13] (also see [14]) are based on central discontinuous Galerkin (DG) methods $[15,16]$ on overlapping Cartesian meshes for nonlinear hyperbolic equations, and use a different discretization for the magnetic induction equations. To be more specific, within each time step, the methods approximate all conservative quantities but the magnetic field with the standard central DG methods. Meanwhile the normal components of the magnetic field on the mesh interfaces are approximated by central DG type methods based on the magnetic induction equations, and this is then followed by an element-by-element divergence-free reconstruction procedure. For higher order accuracy, more information about the magnetic field is extracted, again based on the magnetic induction equations, prior to the reconstruction. The resulting methods in [13] can have arbitrary order of accuracy, and the divergence-free condition of the magnetic field is satisfied exactly up to machine accuracy. Different from Godunov (or upwind) type methods, the methods in [13] are free of exact and approximate Riemann solvers for the MHD system, and they do not explicitly need averaging or interpolation to construct the electric field flux at the grid points.

In this work, we adapt the method in [13] with the discrete space of the lowest order to solve our model problem (1.1), and the forward Euler discretization is applied in time. For this method, we first carry out 
von Neumann analysis on uniform meshes when the velocity field $\mathbf{u}$ is constant, and obtain the time step condition for numerical stability. Though Fourier analysis is simple and straightforward, it can not be applied to more general cases such as equations with variable coefficients or schemes on non-uniform meshes. Motivated by this, we further establish the numerical stability based on energy approach and obtain optimal error estimates for smooth solutions. For the simplicity of the presentation yet without loss of generality, we focus on the energy type analysis when the scheme is applied to the equations with a constant velocity field on uniform meshes (see Appendix for numerical stability when the velocity field is spatially dependent). In the analysis, some non-conventional concepts and techniques are introduced, and this is due to that the method is defined on two overlapping meshes, with some functions defined on mesh interfaces, and involves element-wise reconstruction. This includes a suitably chosen discrete energy which is only associated with the normal component of the magnetic field along mesh interfaces, utilizing the computed solution being exactly divergence-free to establish stability, and identifying the stability mechanisms due to both spatial and temporal discretizations. Approximation properties of the discrete spaces are also used as expected. For the two-dimensional case, the divergence-free condition is closely related to the preservation of the vorticity. In [18], Morton and Roe analyzed a family of vorticity preserving schemes for a system of wave equations based on Fourier analysis for stability as well as local truncation errors of the schemes. For the magnetic induction system itself, there have also been developments based on the related symmetric form of the equations, with some examples including locally divergence-free discontinuous Galerkin methods [4] and finite difference methods [9,17].

The remaining of this paper is organized as follows. In Section 2, the formulation of an exactly divergencefree method is presented. Some properties of the discrete spaces are also discussed. In Section 3, von Neumann analysis is carried out for numerical stability. Following energy methods, in Section 4 we further establish stability analysis and the a prior error estimates. In Section 5, several numerical experiments are presented to confirm or to complement our analytical understanding of the stability condition and the accuracy of the method. Extension of the present work to more general cases is briefly discussed in Section 6 together with some concluding remarks.

\section{Formulation OF THE NUMERICAL METHOD}

In this section, we will introduce some notation and present the formulation of an exactly divergence-free method for the magnetic induction equations (1.1). The computational domain is $\Omega=I \times J=\left[x_{\min }, x_{\max }\right] \times$ $\left[y_{\min }, y_{\max }\right]$, and the boundary conditions are periodic. We use $\phi_{x}$ to denote the $x$ component of a vector-valued function $\phi=\left[\phi_{x}, \phi_{y}\right]^{\top}$, while the $x$-derivative of a function $\psi$ will be $\frac{\partial \psi}{\partial x}$ or $\frac{d \psi}{d x}$. Similar convention will go to the notation with $y$, or possibly with $t$.

\subsection{Meshes and discrete spaces}

Let $\left\{x_{i}\right\}_{i}$ and $\left\{y_{j}\right\}_{j}$ be partitions of $I$ and $J$, respectively, satisfying $x_{\min }=x_{0}<x_{1}<\ldots<x_{N_{x}}=x_{\max }$ and $y_{\min }=y_{0}<y_{1}<\ldots<y_{N_{y}}=y_{\max }$. Let $x_{i+\frac{1}{2}}=\frac{x_{i}+x_{i+1}}{2}$ and $y_{j+\frac{1}{2}}=\frac{y_{j}+y_{j+1}}{2}$. We define two overlapping meshes for $\Omega$, the C-mesh $\mathcal{T}_{h}^{C}=\left\{C_{i, j}, \forall i, j\right\}$ and the D-mesh $\mathcal{T}_{h}^{D}=\left\{D_{i, j}, \forall i, j\right\}$, where $C_{i, j}=I_{i+\frac{1}{2}} \times J_{j+\frac{1}{2}}$ and $D_{i, j}=I_{i} \times J_{j}$, with $I_{i+\frac{1}{2}}=\left[x_{i}, x_{i+1}\right], J_{j+\frac{1}{2}}=\left[y_{j}, y_{j+1}\right], I_{i}=\left[x_{i-\frac{1}{2}}, x_{i+\frac{1}{2}}\right]$ and $J_{j}=\left[y_{j-\frac{1}{2}}, y_{j+\frac{1}{2}}\right]$, see Figure 1 . Without loss of generality, the mesh is assumed to be uniform, with $\left|I_{i}\right|=h_{x}$ and $\left|J_{j}\right|=h_{y}$ (hence $\left|I_{i+\frac{1}{2}}\right|=h_{x}$ and $\left.\left|J_{j+\frac{1}{2}}\right|=h_{y}\right), \forall i, j$, and $h=\max \left(h_{x}, h_{y}\right)$.

To approximate the magnetic field $\mathbf{B}$, we define a finite dimensional discrete space with respect to each mesh,

$$
\begin{aligned}
\mathcal{M}_{h}^{\star} & =\left\{\mathbf{w} \in H\left(\operatorname{div}^{0} ; \Omega\right):\left.\mathbf{w}\right|_{K} \in \mathcal{W}(K), \forall K \in \mathcal{T}_{h}^{\star}, \mathbf{w} \text { is periodic in } \Omega\right\} \\
& =\left\{\mathbf{w} \in\left[L^{2}(\Omega)\right]^{2}:\left.\mathbf{w}\right|_{K} \in \mathcal{W}(K),\left.\nabla \cdot \mathbf{w}\right|_{K}=0, \forall K \in \mathcal{T}_{h}^{\star} \text { and the normal component of } \mathbf{w}\right.
\end{aligned}
$$
is continuous across the mesh interfaces, $\mathbf{w}$ is periodic in $\Omega\}$, 


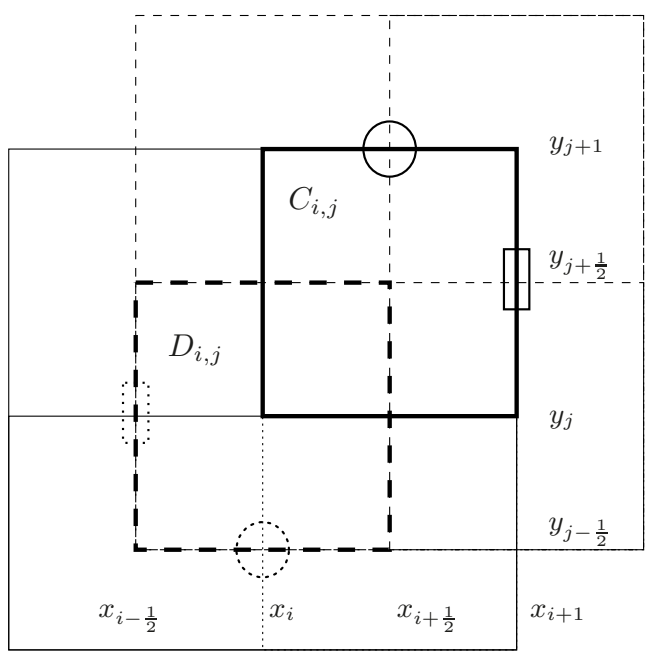

Figure 1. Overlapping meshes in two dimensions. Dotted rectangle: $b_{x, i-\frac{1}{2}, j}^{D} ;$ dotted circle: $b_{y, i, j-\frac{1}{2}}^{D}$; solid rectangle: $b_{x, i+1, j+\frac{1}{2}}^{C}$; solid circle: $b_{y, i+\frac{1}{2}, j+1}^{C}$.

where $H\left(\operatorname{div}^{0} ; \Omega\right)=\{\mathbf{w} \in H(\operatorname{div} ; \Omega): \nabla \cdot \mathbf{w}=0\}$, with $H(\operatorname{div} ; \Omega)=\left\{\mathbf{w} \in\left[L^{2}(\Omega)\right]^{2}: \nabla \cdot \mathbf{w} \in L^{2}(\Omega)\right\}$, and the local space $\mathcal{W}(K)$ is given as

$$
\mathcal{W}(K)=\left\{\mathbf{w} \in\left[L^{2}(K)\right]^{2}: \mathbf{w}=(a, b)^{\top}+c(x,-y)^{\top}, \forall a, b, c \in \mathbb{R}\right\} .
$$

Here and throughout the paper, the superscript $\star$ in the notation (such as $\mathcal{M}_{h}^{\star}$ or $\mathcal{T}_{h}^{\star}$ ) stands for $C$ and $D$. One can see that any function in $\mathcal{M}_{h}^{\star}$ is piecewise-defined linear polynomial, with the normal components being continuous across mesh interfaces, and its divergence being zero.

Next we will summarize some properties of the discrete spaces, and they will be repeatedly used in the analysis. For any $\phi=\left[\phi_{x}, \phi_{y}\right]^{\top} \in \mathcal{W}(K)$, where $K=I_{K} \times J_{K}=\left[x_{l}, x_{r}\right] \times\left[y_{l}, y_{r}\right]$ with the center $(\bar{x}, \bar{y})$, $\left|I_{K}\right|=\Delta x$, and $\left|J_{K}\right|=\Delta y$, then

(i) $\phi_{x}\left(x_{\diamond}, \cdot\right):=\phi_{x}^{\diamond}, \phi_{y}\left(\cdot, y_{\diamond}\right):=\phi_{y}^{\diamond}, \diamond=l, r$, are all constant.

(ii) $\Delta y\left(\phi_{x}^{r}-\phi_{x}^{l}\right)+\Delta x\left(\phi_{y}^{r}-\phi_{y}^{l}\right)=0$.

(iii) $\left.\phi_{x}\right|_{K}=\left(1-\frac{x-x_{l}}{\Delta x}\right) \phi_{x}^{l}+\frac{x-x_{l}}{\Delta x} \phi_{x}^{r},\left.\quad \phi_{y}\right|_{K}=\left(1-\frac{y-y_{l}}{\Delta y}\right) \phi_{y}^{l}+\frac{y-y_{l}}{\Delta y} \phi_{y}^{r}$.

(iv) $\phi_{x}(\bar{x}, \cdot)=\frac{1}{2}\left(\phi_{x}^{l}+\phi_{x}^{r}\right), \quad \phi_{y}(\cdot, \bar{y})=\frac{1}{2}\left(\phi_{y}^{l}+\phi_{y}^{r}\right)$.

(v) With $\|\phi\|_{K}^{2}=\left\|\phi_{x}\right\|_{K}^{2}+\left\|\phi_{y}\right\|_{K}^{2}$, we have

$$
2\|\phi\|_{K}^{2} \leq \Delta x\left(\left\|\phi_{x}\left(x_{l}, \cdot\right)\right\|_{J_{K}}^{2}+\left\|\phi_{x}\left(x_{r}, \cdot\right)\right\|_{J_{K}}^{2}\right)+\Delta y\left(\left\|\phi_{y}\left(\cdot, y_{l}\right)\right\|_{I_{K}}^{2}+\left\|\phi_{y}\left(\cdot, y_{r}\right)\right\|_{I_{K}}^{2}\right) \leq 6\|\phi\|_{K}^{2} .
$$

Here $\|\cdot\|_{K}$ stands for the $L^{2}$ norm on $K$. Similar notation is (will be) used with the subscript $I_{K}, J_{K}, I$, or $J$. The results $(i)-(i v)$ can be verified straightforwardly, with $(i i)$ derived from the divergence-free property. The result in $(v)$ comes from $\left\|\phi_{\diamond}\right\|_{K}^{2}=\frac{1}{3}\left(\left(\phi_{\diamond}^{l}\right)^{2}+\left(\phi_{\diamond}^{r}\right)^{2}+\phi_{\diamond}^{l} \phi_{\diamond}^{r}\right), \diamond=x, y$, directly computed based on (2.2c). From $(i)$, one can see that the normal component of any function in $\mathcal{M}_{h}^{\star}$ on each mesh edge is not only continuous but also constant. The result in $(v)$ implies a new norm for the space $\mathcal{M}_{h}^{\star}$ which is equivalent to the standard $L^{2}$ norm and defined on mesh interfaces (see next lemma). This new norm is more natural to use to analyze our scheme given in Section 2.2. 
Lemma 2.1. For any $\phi^{\star}=\left[\phi_{x}^{\star}, \phi_{y}^{\star}\right]^{\top} \in \mathcal{M}_{h}^{\star}, \star=C, D$, we have

$$
\begin{aligned}
& \left\|\phi^{C}\right\|_{\Omega}^{2} \leq h_{x} \sum_{i}\left\|\phi_{x}^{C}\left(x_{i}, \cdot\right)\right\|_{J}^{2}+h_{y} \sum_{j}\left\|\phi_{y}^{C}\left(\cdot, y_{j}\right)\right\|_{I}^{2} \leq 3\left\|\phi^{C}\right\|_{\Omega}^{2}, \\
& \left\|\phi^{D}\right\|_{\Omega}^{2} \leq h_{x} \sum_{i}\left\|\phi_{x}^{D}\left(x_{i+\frac{1}{2}}, \cdot\right)\right\|_{J}^{2}+h_{y} \sum_{j}\left\|\phi_{y}^{D}\left(\cdot, y_{j+\frac{1}{2}}\right)\right\|_{I}^{2} \leq 3\left\|\phi^{D}\right\|_{\Omega}^{2} .
\end{aligned}
$$

Let $\widetilde{\mathcal{M}}_{h}^{\star}=\left\{\mathbf{w} \in H(\operatorname{div} ; \Omega):\left.\mathbf{w}\right|_{K}=\left(a_{K}+c_{K} x, b_{K}+d_{K} y\right)^{\top}, \forall a_{K}, b_{K}, c_{K}, d_{K} \in \mathbb{R}, \forall K \quad \in\right.$ $\mathcal{T}_{h}^{\star}, \mathbf{w}$ is periodic in $\Omega$ \}. In both the initialization and error analysis, we will use a projection operator $\Pi_{h}^{\star}: H(\operatorname{div} ; \Omega) \mapsto \widetilde{\mathcal{M}}_{h}^{\star}$, which is defined as follows. For any $K \in \mathcal{T}_{h}^{\star}$, with four edges $e_{i}, i=1, \ldots, 4$ and the corresponding outward unit normal $\mathbf{n}_{e_{i}}$, the projection $\Pi_{h}^{\star}$ satisfies

$$
\left(\left(\Pi_{h}^{\star} \phi\right) \cdot \mathbf{n}_{e_{i}}, q\right)_{e_{i}}=\left(\phi \cdot \mathbf{n}_{e_{i}}, q\right)_{e_{i}}, \quad \forall q \in P^{0}\left(e_{i}\right), \quad i=1, \ldots, 4 .
$$

Here $P^{0}\left(e_{i}\right)$ stands for the space defined on $e_{i}$ with constant functions. It is easy to see that the operator $\Pi_{h}^{\star}$ is well defined and it is a projection. It also preserves constant. In fact, the operator can be given explicitly based on its definition. With $\Pi_{h}^{D} \phi$ as an example, we have

$$
\begin{aligned}
& \left.\left(\Pi_{h}^{D} \phi\right)_{x}\right|_{D_{i, j}}=\frac{x-x_{i-\frac{1}{2}}}{h_{x} h_{y}} \int_{y_{j-\frac{1}{2}}}^{y_{j+\frac{1}{2}}}\left(\phi_{x}\left(x_{i+\frac{1}{2}}, y\right)-\phi_{x}\left(x_{i-\frac{1}{2}}, y\right)\right) \mathrm{d} y+\frac{1}{h_{y}} \int_{y_{j-\frac{1}{2}}}^{y_{j+\frac{1}{2}}} \phi_{x}\left(x_{i-\frac{1}{2}}, y\right) \mathrm{d} y, \\
& \left.\left(\Pi_{h}^{D} \phi\right)_{y}\right|_{D_{i, j}}=\frac{y-y_{j-\frac{1}{2}}}{h_{x} h_{y}} \int_{x_{i-\frac{1}{2}}}^{x_{i+\frac{1}{2}}}\left(\phi_{y}\left(x, y_{j+\frac{1}{2}}\right)-\phi_{y}\left(x, y_{j-\frac{1}{2}}\right)\right) \mathrm{d} x+\frac{1}{h_{x}} \int_{x_{i-\frac{1}{2}}}^{x_{i+\frac{1}{2}}} \phi_{y}\left(x, y_{j-\frac{1}{2}}\right) \mathrm{d} x .
\end{aligned}
$$

By using the divergence theorem and the definition of the operator, one can also show

$$
\Pi_{h}^{\star} \phi \in \mathcal{M}_{h}^{\star}, \quad \text { if } \phi \in H\left(\operatorname{div}^{0} ; \Omega\right) .
$$

In other words, $\Pi_{h}^{*}$ defines a projection operator from $H\left(\operatorname{div}^{0} ; \Omega\right)$ to $\mathcal{M}_{h}^{\star}$. In addition, one can establish the following approximation result: there exists a constant $\mathcal{C}>0$, independent of $h$, such that

$$
\left\|\phi-\Pi_{h}^{\star} \phi\right\|_{L^{2}(\Omega)} \leq \mathcal{C}\|\phi\|_{H^{1}(\Omega)} h, \quad \forall \phi \in H(\operatorname{div} ; \Omega) .
$$

This can be proved by using Piola transformation and its properties, the fact of $\Pi_{h}^{\star}$ preserving constant, and the standard scaling argument, see for example (3.17), (3.19), (3.21), (3.31) in [19].

\subsection{An exactly divergence-free method}

In reference [13], a family of exactly divergence-free central discontinuous Galerkin methods of arbitrary order of accuracy was proposed to solve the ideal MHD equations. By assuming the velocity field is given, the methods can be defined directly to solve the magnetic induction equations (1.2). Next, we will formulate such method with the lowest order of accuracy for (1.2). In particular, the method involves central discontinuous Galerkin methods in space defined on mesh edges, followed with an element-by-element reconstruction. In time, the forward Euler method is applied with the time step $\tau$.

We initialize the algorithm at $t=0$ through $\mathbf{B}_{h}^{0, \star}=\Pi_{h}^{\star}\left(\left.\mathbf{B}\right|_{t=0}\right) \in \mathcal{M}_{h}^{\star}$. Given the numerical solutions $\mathbf{B}_{h}^{n, \star}=\left[B_{x, h}^{n, \star}, B_{y, h}^{n, \star}\right]^{\top} \in \mathcal{M}_{h}^{\star}$ at time $t^{n}=n \tau$ with $n \geq 0$, we look for the numerical solutions $\mathbf{B}_{h}^{n+1, \star}=$ $\left[B_{x, h}^{n+1, \star}, B_{y, h}^{n+1, \star}\right]^{\top} \in \mathcal{M}_{h}^{\star}$ at $t^{n+1}=(n+1) \tau$ following two steps. 
Step 1: Look for $\left.b_{x, i}^{n+1, C}(y)\right|_{J_{j+\frac{1}{2}}},\left.b_{y, j}^{n+1, C}(x)\right|_{I_{i+\frac{1}{2}}},\left.b_{x, i+\frac{1}{2}}^{n+1, D}(y)\right|_{J_{j}},\left.b_{y, j+\frac{1}{2}}^{n+1, D}(x)\right|_{I_{i}} \in \mathbb{R}$, such that $\left.\forall \mu_{1}(y)\right|_{J_{j+\frac{1}{2}}}$, $\left.v_{1}(x)\right|_{I_{i+\frac{1}{2}}},\left.\mu_{2}(y)\right|_{J_{j}},\left.v_{2}(x)\right|_{I_{i}} \in \mathbb{R}, \forall i, j$, we have

$$
\left\{\begin{array}{l}
\left(b_{x, i}^{n+1, C}, \mu_{1}\right)_{J_{j+\frac{1}{2}}}=\left(\theta B_{x, h}^{n, D}\left(x_{i}, \cdot\right)+(1-\theta) B_{x, h}^{n, C}\left(x_{i}, \cdot\right), \mu_{1}\right)_{J_{j+\frac{1}{2}}}+\tau G_{j+\frac{1}{2}}\left(E_{z, h}^{n, D}\left(x_{i}, \cdot\right), \mu_{1}\right), \\
\left(b_{y, j}^{n+1, C}, v_{1}\right)_{I_{i+\frac{1}{2}}}=\left(\theta B_{y, h}^{n, D}\left(\cdot, y_{j}\right)+(1-\theta) B_{y, h}^{n, C}\left(\cdot, y_{j}\right), v_{1}\right)_{I_{i+\frac{1}{2}}}+\tau H_{i+\frac{1}{2}}\left(E_{z, h}^{n, D}\left(\cdot, y_{j}\right), v_{1}\right), \\
\left(b_{x, i+\frac{1}{2}}^{n+1, D}, \mu_{2}\right)_{J_{j}}=\left(\theta B_{x, h}^{n, C}\left(x_{i+\frac{1}{2}}, \cdot\right)+(1-\theta) B_{x, h}^{n, D}\left(x_{i+\frac{1}{2}}, \cdot\right), \mu_{2}\right)_{J_{j}}+\tau G_{j}\left(E_{z, h}^{n, C}\left(x_{i+\frac{1}{2}}, \cdot\right), \mu_{2}\right), \\
\left(b_{y, j+\frac{1}{2}}^{n+1, D}, v_{2}\right)_{I_{i}}=\left(\theta B_{y, h}^{n, C}\left(\cdot, y_{j+\frac{1}{2}}\right)+(1-\theta) B_{y, h}^{n, D}\left(\cdot, y_{j+\frac{1}{2}}\right), v_{2}\right)_{I_{i}}+\tau H_{i}\left(E_{z, h}^{n, C}\left(\cdot, y_{j+\frac{1}{2}}\right), v_{2}\right) .
\end{array}\right.
$$

Here $\theta \in[0,1], E_{z, h}^{n, \star}=u_{y} B_{x, h}^{n, \star}-u_{x} B_{y, h}^{n, \star}$, and

$$
\begin{aligned}
G_{j+\frac{1}{2}}(\phi(y), \mu(y)) & =\left(\phi, \frac{\mathrm{d} \mu}{\mathrm{d} y}\right)_{J_{j+\frac{1}{2}}}-\phi\left(y_{j+1}\right) \mu\left(y_{j+1}^{-}\right)+\phi\left(y_{j}\right) \mu\left(y_{j}^{+}\right), \\
G_{j}(\phi(y), \mu(y)) & =\left(\phi, \frac{\mathrm{d} \mu}{\mathrm{d} y}\right)_{J_{j}}-\phi\left(y_{j+\frac{1}{2}}\right) \mu\left(y_{j+\frac{1}{2}}^{-}\right)+\phi\left(y_{j-\frac{1}{2}}\right) \mu\left(y_{j-\frac{1}{2}}^{+}\right), \\
H_{i+\frac{1}{2}}(\phi(x), v(x)) & =-\left(\phi, \frac{\mathrm{d} v}{\mathrm{~d} x}\right)_{I_{i+\frac{1}{2}}}+\phi\left(x_{i+1}\right) v\left(x_{i+1}^{-}\right)-\phi\left(x_{i}\right) v\left(x_{i}^{+}\right), \\
H_{i}(\phi(x), v(x)) & =-\left(\phi, \frac{\mathrm{d} v}{\mathrm{~d} x}\right)_{I_{i}}+\phi\left(x_{i+\frac{1}{2}}\right) v\left(x_{i+\frac{1}{2}}^{-}\right)-\phi\left(x_{i-\frac{1}{2}}\right) v\left(x_{i-\frac{1}{2}}^{+}\right),
\end{aligned}
$$

with $(\cdot, \cdot)_{\mathcal{I}}$ denoting the $L_{2}$ inner product on a bounded domain $\mathcal{I}$, and $\mu\left(y^{ \pm}\right)=\lim _{\epsilon \rightarrow 0^{ \pm}} \mu(y+\epsilon)$. Moreover, given that the test functions in (2.6) are all piecewise constants, the line integrals in (2.7) all vanish.

Step 2: Reconstruct $\mathbf{B}_{h}^{n+1, \star} \in \mathcal{M}_{h}^{\star}$ via an element-by-element procedure. More specifically, on each $C_{i, j}, \forall i, j$, we reconstruct $\left.\mathbf{B}_{h}^{n+1, C}\right|_{C_{i, j}}=\left.\left[B_{x, h}^{n+1, C}, B_{y, h}^{n+1, C}\right]^{\top}\right|_{C_{i, j}} \in \mathcal{W}\left(C_{i, j}\right)$ such that

$$
\begin{aligned}
\text { (i) } B_{x, h}^{n+1, C}\left(x_{\diamond}, y\right) & =b_{x, \diamond}^{n+1, C}(y), \diamond=i, i+1, \forall y \in\left(y_{j}, y_{j+1}\right), \\
\text { (ii) } B_{y, h}^{n+1, C}\left(x, y_{\diamond}\right) & =b_{y, \diamond}^{n+1, C}(x), \diamond=j, j+1, \forall x \in\left(x_{i}, x_{i+1}\right) .
\end{aligned}
$$

Similarly, $\mathbf{B}_{h}^{n+1, D}$ can be defined.

Note that in Step 1 of the algorithm, the normal trace of $\mathbf{B}_{h}^{n+1, \star}$ is first obtained along the mesh edges of $\mathcal{T}_{h}^{\star}$. In Step 2, $\mathbf{B}_{h}^{n+1, \star}$ is reconstructed with an element-by-element procedure. Following the arguments in [13] (see Step 2 of the proof of Thm. 3.1 in [13]), we have the following results for the algorithm regarding the solvability and divergence-free property of the solutions.

Theorem 2.2. Given $\mathbf{B}_{h}^{0, \star} \in \mathcal{M}_{h}^{\star}$, then both $\left.\mathbf{B}_{h}^{n+1, C}\right|_{C_{i j}} \in \mathcal{W}\left(C_{i j}\right)$ and $\left.\mathbf{B}_{h}^{n+1, D}\right|_{D_{i j}} \in \mathcal{W}\left(D_{i j}\right)$ are uniquely determined, $\forall i, j$, hence $\mathbf{B}_{h}^{n+1, \star} \in \mathcal{M}_{h}^{\star}, \forall n \geq 0$.

\section{Numerical Stability By Fourier AnALysis}

In this section, we will establish the numerical stability via Fourier analysis when the meshes are uniform and the velocity field $\mathbf{u}$ is constant. 
On the one hand, we can easily verify that the exact solution to the magnetic induction equations with a constant velocity field, when being divergence-free, satisfies linear advection equation, that is,

$$
\frac{\partial}{\partial t} \mathbf{B}+u_{x} \frac{\partial}{\partial x} \mathbf{B}+u_{y} \frac{\partial}{\partial y} \mathbf{B}=0
$$

a formulation related to the symmetric form of the magnetic induction equations $[4,17]$. When the boundary conditions are periodic, the total energy satisfies

$$
\frac{\mathrm{d}}{\mathrm{d} t} \int_{\Omega}|\mathbf{B}|^{2} \mathrm{~d} x \mathrm{~d} y=0
$$

hence it does not change in time. The numerical stability through Fourier method in this section, and the one based on energy methods in next section (see (4.1)), is a discrete analogue of (3.2). On the other hand, without the divergence-free condition on the magnetic field, magnetic induction equations can not be symmetrized [17] and differ greatly from linear advection equation. Moreover, the system admits solutions growing in time. One such example is given as below when $u_{x}=0$ :

$$
\mathbf{B}(x, y, t)=\left(\sin (x), u_{y} \cos (x) t\right)^{\top} .
$$

Our stability analysis uses the divergence-free property of the computed magnetic field.

Based on Step 2 of the algorithm and the fact that the normal component of $\mathbf{B}_{h}^{n, \star}$ on each edge of the mesh $\mathcal{T}_{h}^{\star}$ is constant, we can introduce the following shorthand notation, $\forall i, j$,

$$
b_{x, i, j+\frac{1}{2}}^{n, C}=\left.b_{x, i}^{n, C}(y)\right|_{J_{j+\frac{1}{2}}}=b_{x, i}^{n, C}\left(y_{j+\frac{1}{2}}\right)=\left.B_{x, h}^{n, C}\left(x_{i}, y\right)\right|_{J_{j+\frac{1}{2}}}=B_{x, h}^{n, C}\left(x_{i}, y_{j+\frac{1}{2}}\right) .
$$

Similarly, we define $b_{y, i+\frac{1}{2}, j}^{n, C}=\left.b_{y, j}^{n, C}(x)\right|_{I_{i+\frac{1}{2}}}=\left.B_{y, h}^{n, C}\left(x, y_{j}\right)\right|_{I_{i+\frac{1}{2}}}, b_{x, i+\frac{1}{2}, j}^{n, D}=\left.b_{x, i+\frac{1}{2}}^{n, D}(y)\right|_{J_{j}}=\left.B_{x, h}^{n, D}\left(x_{i+\frac{1}{2}}, y\right)\right|_{J_{j}}$, and $b_{y, i, j+\frac{1}{2}}^{n, D}=\left.b_{y, j+\frac{1}{2}}^{n, D}(x)\right|_{I_{i}}=\left.B_{y, h}^{n, D}\left(x, y_{j+\frac{1}{2}}\right)\right|_{I_{i}}$, see Figure 1 (without the superscript $n$ ). The proposed scheme now can be written in terms of the new notation. For any $i, j$, and $n \geq 0$,

$$
\begin{aligned}
b_{x, i, j+\frac{1}{2}}^{n+1, C}= & (1-\theta) b_{x, i, j+\frac{1}{2}}^{n, C}+\frac{\tau u_{x}}{2 h_{y}}\left(b_{y, i, j+\frac{3}{2}}^{n, D}-b_{y, i, j-\frac{1}{2}}^{n, D}\right)+\left(\frac{\theta}{4}+\frac{\tau u_{y}}{2 h_{y}}\right)\left(b_{x, i-\frac{1}{2}, j}^{n, D}+b_{x, i+\frac{1}{2}, j}^{n, D}\right) \\
& +\left(\frac{\theta}{4}-\frac{\tau u_{y}}{2 h_{y}}\right)\left(b_{x, i-\frac{1}{2}, j+1}^{n, D}+b_{x, i+\frac{1}{2}, j+1}^{n, D}\right) \\
b_{y, i+\frac{1}{2}, j}^{n+1, C}= & (1-\theta) b_{y, i+\frac{1}{2}, j}^{n, C}+\frac{\tau u_{y}}{2 h_{x}}\left(b_{x, i+\frac{3}{2}, j}^{n, D}-b_{x, i-\frac{1}{2}, j}^{n, D}\right)+\left(\frac{\theta}{4}+\frac{\tau u_{x}}{2 h_{x}}\right)\left(b_{y, i, j-\frac{1}{2}}^{n, D}+b_{y, i, j+\frac{1}{2}}^{n, D}\right) \\
& +\left(\frac{\theta}{4}-\frac{\tau u_{x}}{2 h_{x}}\right)\left(b_{y, i+1, j-\frac{1}{2}}^{n, D}+b_{y, i+1, j+\frac{1}{2}}^{n, D}\right) \\
b_{x, i-\frac{1}{2}, j}^{n+1, D}= & (1-\theta) b_{x, i-\frac{1}{2}, j}^{n, D}+\frac{\tau u_{x}}{2 h_{y}}\left(b_{y, i-\frac{1}{2}, j+1}^{n, C}-b_{y, i-\frac{1}{2}, j-1}^{n, C}\right)+\left(\frac{\theta}{4}+\frac{\tau u_{y}}{2 h_{y}}\right)\left(b_{x, i-1, j-\frac{1}{2}}^{n, C}+b_{x, i, j-\frac{1}{2}}^{n, C}\right) \\
& +\left(\frac{\theta}{4}-\frac{\tau u_{y}}{2 h_{y}}\right)\left(b_{x, i-1, j+\frac{1}{2}}^{n, C}+b_{x, i, j+\frac{1}{2}}^{n, C}\right) \\
b_{y, i, j-\frac{1}{2}}^{n+1, D}= & (1-\theta) b_{y, i, j-\frac{1}{2}}^{n, D}+\frac{\tau u_{y}}{2 h_{x}}\left(b_{x, i+1, j-\frac{1}{2}}^{n, C}-b_{x, i-1, j-\frac{1}{2}}^{n, C}\right)+\left(\frac{\theta}{4}+\frac{\tau u_{x}}{2 h_{x}}\right)\left(b_{y, i-\frac{1}{2}, j-1}^{n, C}+b_{y, i-\frac{1}{2}, j}^{n, C}\right) \\
& +\left(\frac{\theta}{4}-\frac{\tau u_{x}}{2 h_{x}}\right)\left(b_{y, i+\frac{1}{2}, j-1}^{n, C}+b_{y, i+\frac{1}{2}, j}^{n, C}\right) .
\end{aligned}
$$

The divergence-free property of the computed magnetic field also implies the following compatibility conditions,

$$
\left\{\begin{array}{l}
h_{y}\left(b_{x, i+1, j+\frac{1}{2}}^{n, C}-b_{x, i, j+\frac{1}{2}}^{n, C}\right)+h_{x}\left(b_{y, i+\frac{1}{2}, j+1}^{n, C}-b_{y, i+\frac{1}{2}, j}^{n, C}\right)=0 \\
h_{y}\left(b_{x, i+\frac{1}{2}, j}^{n, D}-b_{x, i-\frac{1}{2}, j}^{n, D}\right)+h_{x}\left(b_{y, i, j+\frac{1}{2}}^{n, D}-b_{y, i, j-\frac{1}{2}}^{n, D}\right)=0
\end{array}\right.
$$


Theorem 3.1. For any given $\theta \in(0,1]$, a sufficient and necessary condition for the proposed scheme in (2.6)-(2.8) to be stable is

$$
\left(1-\theta \pm \theta \cos \left(\frac{k_{1} h_{x}}{2}\right) \cos \left(\frac{k_{2} h_{y}}{2}\right)\right)^{2}+\left(\frac{2 \tau u_{x}}{h_{x}} \sin \left(\frac{k_{1} h_{x}}{2}\right) \cos \left(\frac{k_{2} h_{y}}{2}\right)+\frac{2 \tau u_{y}}{h_{y}} \cos \left(\frac{k_{1} h_{x}}{2}\right) \sin \left(\frac{k_{2} h_{y}}{2}\right)\right)^{2} \leq 1
$$

for any $k_{1}, k_{2} \in \mathbb{R}$. Moreover, the scheme is stable under the following CFL condition on the time step $\tau$,

$$
\left(\frac{2 \tau u_{x}}{h_{x}}\right)^{2}+\left(\frac{2 \tau u_{y}}{h_{y}}\right)^{2} \leq \theta
$$

Proof. To carry out the Fourier analysis, we let

$$
\left(b_{x, h}^{n, C}, b_{y, h}^{n, C}, b_{x, h}^{n, D}, b_{y, h}^{n, D}\right)^{\top}=\left(\hat{b}_{x}^{n, C}, \hat{b}_{y}^{n, C}, \hat{b}_{x}^{n, D}, \hat{b}_{y}^{n, D}\right)^{\top} e^{i\left(k_{1} x+k_{2} y\right)}
$$

where $k_{1}, k_{2} \in \mathbb{R}$ being arbitrary. With this ansatz, the scheme (3.3) becomes

$$
\begin{aligned}
& \hat{b}_{x}^{n+1, C}=(1-\theta) \hat{b}_{x}^{n, C}+\frac{\tau u_{x} i}{h_{y}} \sin \left(k_{2} h_{y}\right) \hat{b}_{y}^{n, D}+\cos \left(\frac{k_{1} h_{x}}{2}\right)\left(\theta \cos \left(\frac{k_{2} h_{y}}{2}\right)-\frac{2 \tau u_{y} i}{h_{y}} \sin \left(\frac{k_{2} h_{y}}{2}\right)\right) \hat{b}_{x}^{n, D} \\
& \hat{b}_{y}^{n+1, C}=(1-\theta) \hat{b}_{y}^{n, C}+\frac{\tau u_{y} i}{h_{x}} \sin \left(k_{1} h_{x}\right) \hat{b}_{x}^{n, D}+\cos \left(\frac{k_{2} h_{y}}{2}\right)\left(\theta \cos \left(\frac{k_{1} h_{x}}{2}\right)-\frac{2 \tau u_{x} i}{h_{x}} \sin \left(\frac{k_{1} h_{x}}{2}\right)\right) \hat{b}_{y}^{n, D} \\
& \hat{b}_{x}^{n+1, D}=(1-\theta) \hat{b}_{x}^{n, D}+\frac{\tau u_{x} i}{h_{y}} \sin \left(k_{2} h_{y}\right) \hat{b}_{y}^{n, C}+\cos \left(\frac{k_{1} h_{x}}{2}\right)\left(\theta \cos \left(\frac{k_{2} h_{y}}{2}\right)-\frac{2 \tau u_{y} i}{h_{y}} \sin \left(\frac{k_{2} h_{y}}{2}\right)\right) \hat{b}_{x}^{n, C} \\
& \hat{b}_{y}^{n+1, D}=(1-\theta) \hat{b}_{y}^{n, D}+\frac{\tau u_{y} i}{h_{x}} \sin \left(k_{1} h_{x}\right) \hat{b}_{x}^{n, C}+\cos \left(\frac{k_{2} h_{y}}{2}\right)\left(\theta \cos \left(\frac{k_{1} h_{x}}{2}\right)-\frac{2 \tau u_{x} i}{h_{x}} \sin \left(\frac{k_{1} h_{x}}{2}\right)\right) \hat{b}_{y}^{n, C},
\end{aligned}
$$

while the compatible conditions in (3.4) give

$$
h_{y} \sin \left(\frac{k_{1} h_{x}}{2}\right) \hat{b}_{x}^{n, C}+h_{x} \sin \left(\frac{k_{2} h_{y}}{2}\right) \hat{b}_{y}^{n, C}=0, \quad h_{y} \sin \left(\frac{k_{1} h_{x}}{2}\right) \hat{b}_{x}^{n, D}+h_{x} \sin \left(\frac{k_{2} h_{y}}{2}\right) \hat{b}_{y}^{n, D}=0
$$

Now combining (3.8) and (3.9), one gets

$$
\left\{\begin{array}{l}
\hat{b}_{x}^{n+1, C}=(1-\theta) \hat{b}_{x}^{n, C}+\zeta \hat{b}_{x}^{n, D} \\
\hat{b}_{x}^{n+1, D}=\zeta \hat{b}_{x}^{n, C}+(1-\theta) \hat{b}_{x}^{n, D}
\end{array}\right.
$$

where

$$
\zeta=\theta \cos \left(\frac{k_{1} h_{x}}{2}\right) \cos \left(\frac{k_{2} h_{y}}{2}\right)-\frac{2 \tau u_{x} i}{h_{x}} \sin \left(\frac{k_{1} h_{x}}{2}\right) \cos \left(\frac{k_{2} h_{y}}{2}\right)-\frac{2 \tau u_{y} i}{h_{y}} \cos \left(\frac{k_{1} h_{x}}{2}\right) \sin \left(\frac{k_{2} h_{y}}{2}\right) .
$$

Note that the amplification matrix $\mathcal{Q}$ of $(3.10)$ is a normal matrix, that is, $(\overline{\mathcal{Q}})^{\top} \mathcal{Q}=\mathcal{Q}(\overline{\mathcal{Q}})^{\top}$. This implies a sufficient and necessary condition for the stability of the proposed scheme is that both the eigenvalues of $\mathcal{Q}$ have the magnitude no larger than 1 . More specifically, the two roots $\lambda_{1}$ and $\lambda_{2}$ of the equation $(1-\theta-\lambda)^{2}=\zeta^{2}$ satisfy $\left|\lambda_{1}\right|,\left|\lambda_{2}\right| \leq 1$, and this leads to the condition in (3.5).

Next, we want to obtain a simpler stability condition which can be easier to use in practice. Based on (3.5) and with $\theta \in(0,1]$, a sufficient condition is given below for stability,

$$
\left(\left(\frac{2 \tau u_{x}}{h_{x}}\right)^{2}+\left(\frac{2 \tau u_{y}}{h_{y}}\right)^{2}\right)\left(\xi^{2}\left(1-\eta^{2}\right)+\eta^{2}\left(1-\xi^{2}\right)\right) \leq 1-(1-\theta+\theta \xi \eta)^{2}, \quad \forall \xi, \eta \in[-1,1]
$$


and hence

$$
\left(\left(\frac{2 \tau u_{x}}{h_{x}}\right)^{2}+\left(\frac{2 \tau u_{y}}{h_{y}}\right)^{2}\right)\left(\xi^{2}+\eta^{2}-2(\xi \eta)^{2}\right) \leq \theta\left(-\theta(1-\xi \eta)^{2}+2(1-\xi \eta)\right), \quad \forall \xi, \eta \in[-1,1] .
$$

One can verify that for any fixed $\xi \eta=z \in[-1,1]$ with $\xi, \eta \in[-1,1]$, the maximum value of $\xi^{2}+\eta^{2}$ is $1+z^{2}$. With this, the inequality (3.11) further leads to

$$
\begin{aligned}
& \left(\left(\frac{2 \tau u_{x}}{h_{x}}\right)^{2}+\left(\frac{2 \tau u_{y}}{h_{y}}\right)^{2}\right)\left(1+z^{2}-2 z^{2}\right) \leq \theta\left(-\theta(1-z)^{2}+2(1-z)\right), \quad \forall z \in[-1,1] \\
\Leftrightarrow & \left(\left(\frac{2 \tau u_{x}}{h_{x}}\right)^{2}+\left(\frac{2 \tau u_{y}}{h_{y}}\right)^{2}\right)(1+z) \leq \theta(-\theta(1-z)+2), \quad \forall z \in[-1,1] \\
\Leftrightarrow & \left(\frac{2 \tau u_{x}}{h_{x}}\right)^{2}+\left(\frac{2 \tau u_{y}}{h_{y}}\right)^{2} \leq \theta \min _{z \in[-1,1]} \frac{-\theta(1-z)+2}{1+z}=\theta .
\end{aligned}
$$

This is exactly the sufficient condition (3.6).

\section{NumericAl STABILITY AND ERROR ESTIMATES BY ENERGY METHODS}

The numerical stability established via von Neumann analysis in Section 3 is simple and straightforward, yet the analysis itself only suits for problems with constant coefficients and on uniform meshes. The exactly divergence-free methods in [13] can be defined on non-uniform Cartesian meshes for magnetic induction equations with a variable velocity field as the coefficients, a case which is more relevant to the MHD simulations. Moreover, the methods can be formulated to have $(k+1)$ th order accuracy by using the divergence-free space $\mathcal{M}_{h}^{\star, k}$, a subspace of the $\mathrm{H}(\mathrm{div})$-conforming BDM finite element space (see [13] and also [6]), with any integer $k>0$. (One can see that $\mathcal{M}_{h}^{\star, 0}$ is the same as $\mathcal{M}_{h}^{\star}$ in the present work.) For these methods, a Fourier analysis will boil down to an eigenvalue problem of a $(4 k+2) \times(4 k+2)$ amplification matrix, and such eigenvalue problem in general can not be solved analytically and would rely on numerical computation for any given set of parameters.

Motivated by these considerations, next we will present stability and error estimates based on energy approach, which is seemingly more involved, yet provides a more general framework to analyze the methods without the aforementioned limitations of the Fourier analysis. Though the analysis can be established for general Cartesian meshes and with variable velocity fields, we here only focus on the constant velocity field case on uniform meshes to better illustrate the analysis (see Appendix for numerical stability with a variable velocity field on uniform meshes). The analysis for higher order divergence-free schemes can follow the similar framework, yet with extra complication (potentially) coming from the higher order time discretizations (see e.g. $[23,24]$ for analyzing DG methods with the second and third order Runge-Kutta time discretizations), the additional element-based degrees of freedom to represent the functions in the discrete spaces (see e.g. $[6,13]$ ), or from the need to identify the stability mechanism associated with the use of overlapping meshes. Such analysis will be pursued in a separate project.

\subsection{Stability analysis}

We will start with the stability analysis via energy approach. The main result is given in Theorem 4.1, which is stated in terms of a discrete energy

$$
\mathcal{E}_{h}\left(\mathbf{B}_{h}^{n, C}, \mathbf{B}_{h}^{n, D}\right):=h_{x} \sum_{i}\left(\left\|B_{x, h}^{n, C}\left(x_{i}, \cdot\right)\right\|_{J}^{2}+\left\|B_{x, h}^{n, D}\left(x_{i+\frac{1}{2}}, \cdot\right)\right\|_{J}^{2}\right)+h_{y} \sum_{j}\left(\left\|B_{y, h}^{n, C}\left(\cdot, y_{j}\right)\right\|_{I}^{2}+\left\|B_{y, h}^{n, D}\left(\cdot, y_{j+\frac{1}{2}}\right)\right\|_{I}^{2}\right)
$$

From Lemma 2.1, we know that the square root of this discrete energy is equivalent to the standard $L^{2}$-norm of the numerical solutions, namely, $\left(\left\|\mathbf{B}_{h}^{n, C}\right\|_{\Omega}^{2}+\left\|\mathbf{B}_{h}^{n, D}\right\|_{\Omega}^{2}\right)^{1 / 2}$ for the space $\mathcal{M}_{h}^{C} \times \mathcal{M}_{h}^{D}$. 
Theorem 4.1. Given any $\theta \in(0,1)$, the numerical solutions of the proposed method in (2.6)-(2.8) satisfy

$$
\mathcal{E}_{h}\left(\mathbf{B}_{h}^{n+1, C}, \mathbf{B}_{h}^{n+1, D}\right) \leq \mathcal{E}_{h}\left(\mathbf{B}_{h}^{n, C}, \mathbf{B}_{h}^{n, D}\right), \quad \forall n \geq 0
$$

under the following CFL condition on the time step $\tau$

$$
\tau \leq \tau_{\text {stab }}:=\sqrt{\theta}\left(\frac{1}{2}-\frac{\sqrt{\theta}}{2}\right) \sqrt{\frac{\min \left(h_{x}, h_{y}\right) h_{x} h_{y}}{\max \left(\left|u_{x}\right|,\left|u_{y}\right|\right)\left(\left|u_{x}\right|+\left|u_{y}\right|\right)\left(h_{x}+h_{y}\right)}} .
$$

To make the proof of Theorem 4.1 easier to follow, we next organize the technical details into some preparatory lemmas in Section 4.1.1, whose proofs can be skipped by the readers during their first round of reading, while the main stability result is proved in Section 4.1.2.

\subsubsection{Preliminary lemmas}

For all the lemmas below, the results greatly rely on the properties of the discrete spaces summarized in (2.2). In particular, to get Lemma 4.3, one needs the approximating functions to be divergence-free.

We will soon see that there are two types of terms contributing to numerical stability. The first type is related to $\mathbf{B}_{h}^{n+1, \star}-\mathbf{B}_{h}^{n, \star}$, particularly in the form of

$$
\begin{array}{ll}
\sum_{i}\left\|B_{x, h}^{n+1, D}\left(x_{i+\frac{1}{2}}, \cdot\right)-B_{x, h}^{n, D}\left(x_{i+\frac{1}{2}}, \cdot\right)\right\|_{J}^{2}, & \sum_{i}\left\|B_{x, h}^{n+1, C}\left(x_{i}, \cdot\right)-B_{x, h}^{n, C}\left(x_{i}, \cdot\right)\right\|_{J}^{2}, \\
\sum_{j}\left\|B_{y, h}^{n+1, D}\left(\cdot, y_{j+\frac{1}{2}}\right)-B_{y, h}^{n, D}\left(\cdot, y_{j+\frac{1}{2}}\right)\right\|_{I}^{2}, & \sum_{j}\left\|B_{y, h}^{n+1, C}\left(\cdot, y_{j}\right)-B_{y, h}^{n, C}\left(\cdot, y_{j}\right)\right\|_{I}^{2},
\end{array}
$$

and such terms are related to the use of the forward (or backward) Euler method in time. The other type is more unique for the central methods on overlapping meshes. For the method proposed here, the terms are in the form of

$$
\sum_{i} \sum_{s=i \pm \frac{1}{2}}\left\|B_{x, h}^{n, D}\left(x_{s}, \cdot\right)-B_{x, h}^{n, C}\left(x_{i}, \cdot\right)\right\|_{J}^{2}, \quad \sum_{j} \sum_{s=j \pm \frac{1}{2}}\left\|B_{y, h}^{n, D}\left(\cdot, y_{s}\right)-B_{y, h}^{n, C}\left(\cdot, y_{j}\right)\right\|_{I}^{2}
$$

These terms serve a similar role as the jumps of the approximating functions at the mesh interfaces in the analysis of discontinuous Galerkin methods [7]. In the proof of Lemma 4.4, a crucial ingredient is to properly decompose some expressions into terms in (4.3). Similar as in Section 3, the following shorthand notation will be used in this subsection. For any given $\phi^{\star}=\left[\phi_{x}^{\star}, \phi_{y}^{\star}\right]^{\top} \in \mathcal{M}_{h}^{\star}, \star=C$ or $D$, and with $\forall i, j$, we let

$$
\phi_{x, i, j+\frac{1}{2}}^{C}=\left.\phi_{x}^{C}\left(x_{i}, y\right)\right|_{J_{j+\frac{1}{2}}}, \phi_{y, i+\frac{1}{2}, j}^{C}=\left.\phi_{y}^{C}\left(x, y_{j}\right)\right|_{I_{i+\frac{1}{2}}}, \phi_{x, i+\frac{1}{2}, j}^{D}=\left.\phi_{x}^{D}\left(x_{i+\frac{1}{2}}, y\right)\right|_{J_{j}}, \phi_{y, i, j+\frac{1}{2}}^{D}=\left.\phi_{y}^{D}\left(x, y_{j+\frac{1}{2}}\right)\right|_{I_{i}},
$$

see Figure 1, with $b$ in the plot replaced by $\phi$.

Lemma 4.2. Let $\varphi^{C}=\left[\varphi_{x}^{C}, \varphi_{y}^{C}\right]^{\top} \in \mathcal{M}_{h}^{C}, \phi^{D}=\left[\phi_{x}^{D}, \phi_{y}^{D}\right]^{\top} \in \mathcal{M}_{h}^{D}$, then

$$
\begin{aligned}
& \sum_{i}\left(\phi_{x}^{D}\left(x_{i}, \cdot\right)-\varphi_{x}^{C}\left(x_{i}, \cdot\right), \varphi_{x}^{C}\left(x_{i}, \cdot\right)\right)_{J}+\sum_{i}\left(\varphi_{x}^{C}\left(x_{i+\frac{1}{2}}, \cdot\right)-\phi_{x}^{D}\left(x_{i+\frac{1}{2}}, \cdot\right), \phi_{x}^{D}\left(x_{i+\frac{1}{2}}, \cdot\right)\right)_{J} \\
= & -\frac{1}{2} \sum_{i} \sum_{s=i \pm \frac{1}{2}}\left\|\phi_{x}^{D}\left(x_{s}, \cdot\right)-\varphi_{x}^{C}\left(x_{i}, \cdot\right)\right\|_{J}^{2} .
\end{aligned}
$$


Proof. Using the property of $\mathcal{W}(K)$ in $(2.2 \mathrm{~d})$, and the periodicity of the involved functions, one gets

$$
\begin{aligned}
& \sum_{i}\left(\phi_{x}^{D}\left(x_{i}, \cdot\right)-\varphi_{x}^{C}\left(x_{i}, \cdot\right), \varphi_{x}^{C}\left(x_{i}, \cdot\right)\right)_{J}+\sum_{i}\left(\varphi_{x}^{C}\left(x_{i+\frac{1}{2}}, \cdot\right)-\phi_{x}^{D}\left(x_{i+\frac{1}{2}}, \cdot\right), \phi_{x}^{D}\left(x_{i+\frac{1}{2}}, \cdot\right)\right)_{J} \\
= & \frac{1}{2} \sum_{i}\left(\phi_{x}^{D}\left(x_{i-\frac{1}{2}}, \cdot\right)-\varphi_{x}^{C}\left(x_{i}, \cdot\right), \varphi_{x}^{C}\left(x_{i}, \cdot\right)\right)_{J}+\frac{1}{2} \sum_{i}\left(\phi_{x}^{D}\left(x_{i+\frac{1}{2}}, \cdot\right)-\varphi_{x}^{C}\left(x_{i}, \cdot\right), \varphi_{x}^{C}\left(x_{i}, \cdot\right)\right)_{J} \\
& +\frac{1}{2} \sum_{i}\left(\varphi_{x}^{C}\left(x_{i}, \cdot\right)-\phi_{x}^{D}\left(x_{i+\frac{1}{2}}, \cdot\right), \phi_{x}^{D}\left(x_{i+\frac{1}{2}}, \cdot\right)\right)_{J}+\frac{1}{2} \sum_{i}\left(\varphi_{x}^{C}\left(x_{i+1}, \cdot\right)-\phi_{x}^{D}\left(x_{i+\frac{1}{2}}, \cdot\right), \phi_{x}^{D}\left(x_{i+\frac{1}{2}}, \cdot\right)\right)_{J} \\
= & -\frac{1}{2} \sum_{i}\left\|\phi_{x}^{D}\left(x_{i+\frac{1}{2}}, \cdot\right)-\varphi_{x}^{C}\left(x_{i+1}, \cdot\right)\right\|_{J}^{2}-\frac{1}{2} \sum_{i}\left\|\phi_{x}^{D}\left(x_{i+\frac{1}{2}}, \cdot\right)-\varphi_{x}^{C}\left(x_{i}, \cdot\right)\right\|_{J}^{2} \cdot
\end{aligned}
$$

Lemma 4.3. Let $\varphi^{C}=\left[\varphi_{x}^{C}, \varphi_{y}^{C}\right]^{\top} \in \mathcal{M}_{h}^{C}, \phi^{D}=\left[\phi_{x}^{D}, \phi_{y}^{D}\right]^{\top} \in \mathcal{M}_{h}^{D}$, then

$$
\begin{aligned}
& \text { (i) } \sum_{i, j} G_{j+\frac{1}{2}}\left(\phi_{x}^{D}\left(x_{i}, \cdot\right), \varphi_{x}^{C}\left(x_{i}, \cdot\right)\right)+\sum_{i, j} G_{j}\left(\varphi_{x}^{C}\left(x_{i+\frac{1}{2}}, \cdot\right), \phi_{x}^{D}\left(x_{i+\frac{1}{2}}, \cdot\right)\right)=0, \\
& \text { (ii) } \sum_{i, j} G_{j+\frac{1}{2}}\left(\phi_{y}^{D}\left(x_{i}, \cdot\right), \varphi_{x}^{C}\left(x_{i}, \cdot\right)\right)+\sum_{i, j} G_{j}\left(\varphi_{y}^{C}\left(x_{i+\frac{1}{2}}, \cdot\right), \phi_{x}^{D}\left(x_{i+\frac{1}{2}}, \cdot\right)\right)=0 .
\end{aligned}
$$

Proof. To prove equality (4.6), we first rewrite $G_{j+\frac{1}{2}}\left(\phi_{x}^{D}\left(x_{i}, \cdot\right), \varphi_{x}^{C}\left(x_{i}, \cdot\right)\right)$ and $G_{j}\left(\varphi_{x}^{C}\left(x_{i+\frac{1}{2}}, \cdot\right), \phi_{x}^{D}\left(x_{i+\frac{1}{2}}, \cdot\right)\right)$ based on the properties of $\mathcal{W}(K)$ in $(2.2 \mathrm{~d})$ and $(2.2 \mathrm{a})$,

$$
\begin{aligned}
G_{j+\frac{1}{2}}\left(\phi_{x}^{D}\left(x_{i}, \cdot\right), \varphi_{x}^{C}\left(x_{i}, \cdot\right)\right) & =\frac{1}{2}\left(-\phi_{x, i-\frac{1}{2}, j+1}^{D}-\phi_{x, i+\frac{1}{2}, j+1}^{D}+\phi_{x, i-\frac{1}{2}, j}^{D}+\phi_{x, i+\frac{1}{2}, j}^{D}\right) \varphi_{x, i, j+\frac{1}{2}}^{C}, \\
G_{j}\left(\varphi_{x}^{C}\left(x_{i+\frac{1}{2}}, \cdot\right), \phi_{x}^{D}\left(x_{i+\frac{1}{2}}, \cdot\right)\right) & =\frac{1}{2}\left(-\varphi_{x, i, j+\frac{1}{2}}^{C}-\varphi_{x, i+1, j+\frac{1}{2}}^{C}+\varphi_{x, i, j-\frac{1}{2}}^{C}+\varphi_{x, i+1, j-\frac{1}{2}}^{C}\right) \phi_{x, i+\frac{1}{2}, j}^{D} .
\end{aligned}
$$

Summing them up over $i, j$, and rearranging the sub-indices, we obtain (4.6).

To prove (4.7), we again rewrite $G_{j+\frac{1}{2}}\left(\phi_{y}^{D}\left(x_{i}, \cdot\right), \varphi_{x}^{C}\left(x_{i}, \cdot\right)\right)$ based on the property of $\mathcal{W}(K)$ in $(2.2 \mathrm{~d})$ and $(2.2 \mathrm{a})$, in addition, we will utilize the divergence-free property of $\phi^{D}$ given in (2.2b) to reformulate $\phi_{y}^{D}$ in terms of $\phi_{x}^{D}$,

$$
\begin{aligned}
G_{j+\frac{1}{2}}\left(\phi_{y}^{D}\left(x_{i}, \cdot\right), \varphi_{x}^{C}\left(x_{i}, \cdot\right)\right) & =\frac{1}{2}\left(-\phi_{y, i, j+\frac{1}{2}}^{D}-\phi_{y, i, j+\frac{3}{2}}^{D}+\phi_{y, i, j-\frac{1}{2}}^{D}+\phi_{y, i, j+\frac{1}{2}}^{D}\right) \varphi_{x, i, j+\frac{1}{2}}^{C} \\
& =\frac{h_{y}}{2 h_{x}}\left(\phi_{x, i+\frac{1}{2}, j}^{D}-\phi_{x, i-\frac{1}{2}, j}^{D}+\phi_{x, i+\frac{1}{2}, j+1}^{D}-\phi_{x, i-\frac{1}{2}, j+1}^{D}\right) \varphi_{x, i, j+\frac{1}{2}}^{C} .
\end{aligned}
$$

Similarly,

$$
G_{j}\left(\varphi_{y}^{C}\left(x_{i+\frac{1}{2}}, \cdot\right), \phi_{x}^{D}\left(x_{i+\frac{1}{2}}, \cdot\right)\right)=\frac{h_{y}}{2 h_{x}}\left(\varphi_{x, i+1, j-\frac{1}{2}}^{C}-\varphi_{x, i, j-\frac{1}{2}}^{C}+\varphi_{x, i+1, j+\frac{1}{2}}^{C}-\varphi_{x, i, j+\frac{1}{2}}^{C}\right) \phi_{x, i+\frac{1}{2}, j}^{D} .
$$

Summing (4.11) and (4.12) up over $i$ and $j$, together with the periodicity, we get (4.7).

Lemma 4.4. Let $\phi^{\star}=\left[\phi_{x}^{\star}, \phi_{y}^{\star}\right]^{\top}, \varphi^{\star}=\left[\varphi_{x}^{\star}, \varphi_{y}^{\star}\right]^{\top} \in \mathcal{M}_{h}^{\star}$, then for any $\alpha>0$, we have

(i) $\sum_{i, j} G_{j+\frac{1}{2}}\left(\phi_{x}^{D}\left(x_{i}, \cdot\right), \varphi_{x}^{C}\left(x_{i}, \cdot\right)\right) \leq \frac{\alpha}{h_{y}} \sum_{i}\left\|\varphi_{x}^{C}\left(x_{i}, \cdot\right)\right\|_{J}^{2}+\frac{1}{2 \alpha h_{y}} \sum_{i} \sum_{s=i \pm \frac{1}{2}}\left\|\phi_{x}^{D}\left(x_{s}, \cdot\right)-\phi_{x}^{C}\left(x_{i}, \cdot\right)\right\|_{J}^{2}$,

(ii) $\sum_{i, j} G_{j+\frac{1}{2}}\left(\phi_{y}^{D}\left(x_{i}, \cdot\right), \varphi_{x}^{C}\left(x_{i}, \cdot\right)\right) \leq \frac{\alpha}{h_{y}} \sum_{i}\left\|\varphi_{x}^{C}\left(x_{i}, \cdot\right)\right\|_{J}^{2}+\frac{1}{2 \alpha h_{x}} \sum_{j} \sum_{s=j \pm \frac{1}{2}}\left\|\phi_{y}^{D}\left(\cdot, y_{s}\right)-\phi_{y}^{C}\left(\cdot, y_{j}\right)\right\|_{I}^{2}$, 
(iii) $\sum_{i, j} G_{j}\left(\phi_{x}^{C}\left(x_{i+\frac{1}{2}}, \cdot\right), \varphi_{x}^{D}\left(x_{i+\frac{1}{2}}, \cdot\right)\right) \leq \frac{\alpha}{h_{y}} \sum_{i}\left\|\varphi_{x}^{D}\left(x_{i+\frac{1}{2}}, \cdot\right)\right\|_{J}^{2}+\frac{1}{2 \alpha h_{y}} \sum_{i} \sum_{s=i \pm \frac{1}{2}}\left\|\phi_{x}^{D}\left(x_{s}, \cdot\right)-\phi_{x}^{C}\left(x_{i}, \cdot\right)\right\|_{J}^{2}$,

(iv) $\sum_{i, j} G_{j}\left(\phi_{y}^{C}\left(x_{i+\frac{1}{2}}, \cdot\right), \varphi_{x}^{D}\left(x_{i+\frac{1}{2}}, \cdot\right)\right) \leq \frac{\alpha}{h_{y}} \sum_{i}\left\|\varphi_{x}^{D}\left(x_{i+\frac{1}{2}}, \cdot\right)\right\|_{J}^{2}+\frac{1}{2 \alpha h_{x}} \sum_{j} \sum_{s=j \pm \frac{1}{2}}\left\|\phi_{y}^{D}\left(\cdot, y_{s}\right)-\phi_{y}^{C}\left(\cdot, y_{j}\right)\right\|_{I}^{2}$.

Proof. Based on (4.8), we can derive the result in part (i) by properly inserting and subtracting some terms defined on $C$-mesh, followed with the use of Young's inequality and the property of $\mathcal{W}(K)$ in $(2.2 \mathrm{a})$.

$$
\begin{aligned}
\sum_{i, j} G_{j+\frac{1}{2}}\left(\phi_{x}^{D}\left(x_{i}, \cdot\right), \varphi_{x}^{C}\left(x_{i}, \cdot\right)\right)= & \frac{1}{2} \sum_{i, j}\left(-\phi_{x, i-\frac{1}{2}, j+1}^{D}-\phi_{x, i+\frac{1}{2}, j+1}^{D}+\phi_{x, i-\frac{1}{2}, j}^{D}+\phi_{x, i+\frac{1}{2}, j}^{D}\right) \varphi_{x, i, j+\frac{1}{2}}^{C} \\
\leq & \frac{1}{4 \alpha} \sum_{i, j}\left(\left(\phi_{x, i-\frac{1}{2}, j+1}^{D}-\phi_{x, i, j+\frac{1}{2}}^{C}\right)^{2}+\left(\phi_{x, i+\frac{1}{2}, j+1}^{D}-\phi_{x, i, j+\frac{1}{2}}^{C}\right)^{2}\right)+\alpha \sum_{i, j}\left(\varphi_{x, i, j+\frac{1}{2}}^{C}\right)^{2} \\
& +\frac{1}{4 \alpha} \sum_{i, j}\left(\left(\phi_{x, i-\frac{1}{2}, j}^{D}-\phi_{x, i, j+\frac{1}{2}}^{C}\right)^{2}+\left(\phi_{x, i+\frac{1}{2}, j}^{D}-\phi_{x, i, j+\frac{1}{2}}^{C}\right)^{2}\right) \\
= & \frac{1}{2 \alpha h_{y}} \sum_{i}\left(\left\|\phi_{x}^{D}\left(x_{i-\frac{1}{2}}, \cdot\right)-\phi_{x}^{C}\left(x_{i}, \cdot\right)\right\|_{J}^{2}+\left\|\phi_{x}^{D}\left(x_{i+\frac{1}{2}}, \cdot\right)-\phi_{x}^{C}\left(x_{i}, \cdot\right)\right\|_{J}^{2}\right) \\
& +\frac{\alpha}{h_{y}} \sum_{i}\left\|\varphi_{x}^{C}\left(x_{i}, \cdot\right)\right\|_{J}^{2} .
\end{aligned}
$$

To establish the result in part (ii), we start with (4.10),

$$
\sum_{i, j} G_{j+\frac{1}{2}}\left(\phi_{y}^{D}\left(x_{i}, y\right), \varphi_{x}^{C}\left(x_{i}, y\right)\right)=-\frac{1}{2}\left(\phi_{y, i, j+\frac{3}{2}}^{D}-\phi_{y, i, j-\frac{1}{2}}^{D}\right) \varphi_{x, i, j+\frac{1}{2}}^{C}
$$

and rewrite $\phi_{y, i, j+\frac{3}{2}}^{D}-\phi_{y, i, j-\frac{1}{2}}^{D}$ by properly inserting and subtracting some terms on $C$-mesh,

$$
\phi_{y, i, j+\frac{3}{2}}^{D}-\phi_{y, i, j-\frac{1}{2}}^{D}=\phi_{y, i, j+\frac{3}{2}}^{D} \mp \phi_{y, i+\frac{1}{2}, j+1}^{C} \mp \phi_{y, i, j+\frac{1}{2}}^{D} \mp \phi_{y, i+\frac{1}{2}, j}^{C}-\phi_{y, i, j-\frac{1}{2}}^{D} .
$$

Now using (4.14), Young's inequality, and the property (2.2a) of $\mathcal{W}(K)$, we can get

$$
\begin{aligned}
& \sum_{i, j} G_{j+\frac{1}{2}}\left(\phi_{y}^{D}\left(x_{i}, y\right), \varphi_{x}^{C}\left(x_{i}, y\right)\right) \\
\leq & \frac{1}{4 \alpha} \sum_{i, j}\left(\left(\phi_{y, i, j+\frac{3}{2}}^{D}-\phi_{y, i+\frac{1}{2}, j+1}^{C}\right)^{2}+\left(\phi_{y, i+\frac{1}{2}, j+1}^{C}-\phi_{y, i, j+\frac{1}{2}}^{D}\right)^{2}\right) \\
& +\frac{1}{4 \alpha} \sum_{i, j}\left(\left(\phi_{y, i, j+\frac{1}{2}}^{D}-\phi_{y, i+\frac{1}{2}, j}^{C}\right)^{2}+\left(\phi_{y, i+\frac{1}{2}, j}^{C}-\phi_{y, i, j-\frac{1}{2}}^{D}\right)^{2}\right)+\alpha \sum_{i}\left(\varphi_{x, i, j+\frac{1}{2}}^{C}\right)^{2} \\
= & \frac{1}{2 \alpha h_{x}} \sum_{j}\left(\left\|\phi_{y}^{D}\left(\cdot, y_{j+\frac{1}{2}}\right)-\phi_{y}^{C}\left(\cdot, y_{j}\right)\right\|_{I}^{2}+\left\|\phi_{y}^{D}\left(\cdot, y_{j+\frac{1}{2}}\right)-\phi_{y}^{C}\left(\cdot, y_{j+1}\right)\right\|_{I}^{2}\right)+\frac{\alpha}{h_{y}} \sum_{i}\left\|\varphi_{x}^{C}\left(x_{i}, \cdot\right)\right\|_{J}^{2} .
\end{aligned}
$$

This completes part (ii) of the lemma. (iii) and (iv) can be proved similarly. 


\subsubsection{Stability analysis: proof of Theorem 4.1}

Taking $\mu_{1}(y)=b_{x, i}^{n+1, C}(y)=B_{x, h}^{n+1, C}\left(x_{i}, y\right), \mu_{2}(y)=b_{x, i+\frac{1}{2}}^{n+1, D}(y)=B_{x, h}^{n+1, D}\left(x_{i+\frac{1}{2}}, y\right)$ in (2.6), and summing up the resulting equations over all $i, j$, we obtain

$$
\begin{aligned}
\frac{1}{2} \sum_{i}\left(\left\|b_{x, i}^{n+1, C}\right\|_{J}^{2}+\left\|b_{x, i+\frac{1}{2}}^{n+1, D}\right\|_{J}^{2}-\left\|b_{x, i}^{n, C}\right\|_{J}^{2}-\left\|b_{x, i+\frac{1}{2}}^{n, D}\right\|_{J}^{2}\right)= & -\frac{1}{2} \sum_{i}\left\|B_{x, h}^{n+1, C}\left(x_{i}, \cdot\right)-B_{x, h}^{n, C}\left(x_{i}, \cdot\right)\right\|_{J}^{2} \\
& -\frac{1}{2} \sum_{i}\left\|B_{x, h}^{n+1, D}\left(x_{i+\frac{1}{2}}, \cdot\right)-B_{x, h}^{n, D}\left(x_{i+\frac{1}{2}}, \cdot\right)\right\|_{J}^{2} \\
& +\Lambda_{1}+\Lambda_{2}+\Lambda_{3}+\Lambda_{4},
\end{aligned}
$$

where

$$
\begin{aligned}
\Lambda_{1}= & \theta \sum_{i}\left\{\left(B_{x, h}^{n, D}\left(x_{i}, \cdot\right)-B_{x, h}^{n, C}\left(x_{i}, \cdot\right), B_{x, h}^{n, C}\left(x_{i}, \cdot\right)\right)_{J}+\left(B_{x, h}^{n, C}\left(x_{i+\frac{1}{2}}, \cdot\right)-B_{x, h}^{n, D}\left(x_{i+\frac{1}{2}}, \cdot\right), B_{x, h}^{n, D}\left(x_{i+\frac{1}{2}}, \cdot\right)\right)_{J}\right\}, \\
\Lambda_{2}= & \tau \sum_{i, j} G_{j+\frac{1}{2}}\left(E_{z, h}^{n, D}\left(x_{i}, \cdot\right), B_{x, h}^{n, C}\left(x_{i}, \cdot\right)\right)+\tau \sum_{i, j} G_{j}\left(E_{z, h}^{n, C}\left(x_{i+\frac{1}{2}}, \cdot\right), B_{x, h}^{n, D}\left(x_{i+\frac{1}{2}}, \cdot\right)\right), \\
\Lambda_{3}= & \theta \sum_{i}\left(B_{x, h}^{n, D}\left(x_{i}, \cdot\right)-B_{x, h}^{n, C}\left(x_{i}, \cdot\right), B_{x, h}^{n+1, C}\left(x_{i}, \cdot\right)-B_{x, h}^{n, C}\left(x_{i}, \cdot\right)\right)_{J} \\
& +\theta \sum_{i}\left(B_{x, h}^{n, C}\left(x_{i+\frac{1}{2}}, \cdot\right)-B_{x, h}^{n, D}\left(x_{i+\frac{1}{2}}, \cdot\right), B_{x, h}^{n+1, D}\left(x_{i+\frac{1}{2}}, \cdot\right)-B_{x, h}^{n, D}\left(x_{i+\frac{1}{2}}, \cdot\right)\right)_{J}, \\
\Lambda_{4}= & \tau \sum_{i, j} G_{j+\frac{1}{2}}\left(E_{z, h}^{n, D}\left(x_{i}, \cdot\right), B_{x, h}^{n+1, C}\left(x_{i}, \cdot\right)-B_{x, h}^{n, C}\left(x_{i}, \cdot\right)\right) \\
& +\tau \sum_{i, j} G_{j}\left(E_{z, h}^{n, C}\left(x_{i+\frac{1}{2}}, \cdot\right), B_{x, h}^{n+1, D}\left(x_{i+\frac{1}{2}}, \cdot\right)-B_{x, h}^{n, D}\left(x_{i+\frac{1}{2}}, \cdot\right)\right) .
\end{aligned}
$$

Next we estimate each $\Lambda_{i}$ term, $i=1, \ldots, 4$. For $\Lambda_{1}$, with $\mathbf{B}_{h}^{n, \star} \in \mathcal{M}_{h}^{\star}$, we take $\varphi^{C}=\mathbf{B}_{h}^{n, C}, \phi^{D}=\mathbf{B}_{h}^{n, D}$ in Lemma 4.2 and obtain

$$
\Lambda_{1}=-\frac{\theta}{2} \sum_{i} \sum_{s=i \pm \frac{1}{2}}\left\|B_{x, h}^{n, D}\left(x_{s}, \cdot\right)-B_{x, h}^{n, C}\left(x_{i}, \cdot\right)\right\|_{J}^{2} .
$$

For $\Lambda_{2}$, using $E_{z, h}^{n, \star}=u_{y} B_{x, h}^{n, \star}-u_{x} B_{y, h}^{n, \star}$, the bilinearity of $G_{j}, G_{j+\frac{1}{2}}$, and taking $\varphi^{C}=\mathbf{B}_{h}^{n, C}$ and $\phi^{D}=\mathbf{B}_{h}^{n, D}$ in Lemma 4.3, we have

$$
\Lambda_{2}=0
$$

With (2.2d) and Young's inequality, we can estimate $\Lambda_{3}$ with any $\alpha_{1}>0$ as follows.

$$
\begin{aligned}
\Lambda_{3} \leq & \frac{\theta^{2}}{4 \alpha_{1}} \sum_{i}\left\|\frac{1}{2} B_{x, h}^{n, D}\left(x_{i-\frac{1}{2}}, \cdot\right)+\frac{1}{2} B_{x, h}^{n, D}\left(x_{i+\frac{1}{2}}, \cdot\right)-B_{x, h}^{n, C}\left(x_{i}, \cdot\right)\right\|_{J}^{2} \\
& +\frac{\theta^{2}}{4 \alpha_{1}} \sum_{i}\left\|B_{x, h}^{n, D}\left(x_{i+\frac{1}{2}}, \cdot\right)-\frac{1}{2} B_{x, h}^{n, C}\left(x_{i}, \cdot\right)-\frac{1}{2} B_{x, h}^{n, C}\left(x_{i+1}, \cdot\right)\right\|_{J}^{2} \\
& +\alpha_{1} \sum_{i}\left(\left\|B_{x, h}^{n+1, C}\left(x_{i}, \cdot\right)-B_{x, h}^{n, C}\left(x_{i}, \cdot\right)\right\|_{J}^{2}+\left\|B_{x, h}^{n+1, D}\left(x_{i+\frac{1}{2}}, \cdot\right)-B_{x, h}^{n, D}\left(x_{i+\frac{1}{2}}, \cdot\right)\right\|_{J}^{2}\right) \\
\leq & \frac{\theta^{2}}{4 \alpha_{1}} \sum_{i} \sum_{s=i \pm \frac{1}{2}}\left\|B_{x, h}^{n, D}\left(x_{s}, \cdot\right)-B_{x, h}^{n, C}\left(x_{i}, \cdot\right)\right\|_{J}^{2} \\
& +\alpha_{1} \sum_{i}\left(\left\|B_{x, h}^{n+1, C}\left(x_{i}, \cdot\right)-B_{x, h}^{n, C}\left(x_{i}, \cdot\right)\right\|_{J}^{2}+\left\|B_{x, h}^{n+1, D}\left(x_{i+\frac{1}{2}}, \cdot\right)-B_{x, h}^{n, D}\left(x_{i+\frac{1}{2}}, \cdot\right)\right\|_{J}^{2}\right) .
\end{aligned}
$$

The last inequality is obtained by applying $(a+b)^{2} \leq 2\left(a^{2}+b^{2}\right)$ and re-arranging the indices. 
For the last term $\Lambda_{4}$, using $E_{z, h}^{n, \star}=u_{y} B_{x, h}^{n, \star}-u_{x} B_{y, h}^{n, \star}$, the bilinearity of $G_{j}, G_{j+\frac{1}{2}}$, and applying (i)-(iv) of Lemma 4.4 with $\forall \alpha_{2}>0$, we have

$$
\begin{aligned}
\Lambda_{4} \leq & \frac{\alpha_{2}\left(\left|u_{x}\right|+\left|u_{y}\right|\right) \tau}{h_{y}} \sum_{i}\left(\left\|B_{x, h}^{n+1, D}\left(x_{i+\frac{1}{2}}, \cdot\right)-B_{x, h}^{n, D}\left(x_{i+\frac{1}{2}}, \cdot\right)\right\|_{J}^{2}+\left\|B_{x, h}^{n+1, C}\left(x_{i}, \cdot\right)-B_{x, h}^{n, C}\left(x_{i}, \cdot\right)\right\|_{J}^{2}\right) \\
& +\frac{\left|u_{y}\right| \tau}{\alpha_{2} h_{y}} \sum_{i} \sum_{s=i \pm \frac{1}{2}}\left\|B_{x, h}^{n, D}\left(x_{s}, \cdot\right)-B_{x, h}^{n, C}\left(x_{i}, \cdot\right)\right\|_{J}^{2}+\frac{\left|u_{x}\right| \tau}{\alpha_{2} h_{x}} \sum_{j} \sum_{s=j \pm \frac{1}{2}}\left\|B_{y, h}^{n, D}\left(\cdot, y_{s}\right)-B_{y, h}^{n, C}\left(\cdot, y_{j}\right)\right\|_{I}^{2} .
\end{aligned}
$$

Now we can sum up (4.16)-(4.19), and provide an upper bound for (4.15),

$$
\begin{aligned}
& \frac{1}{2} \sum_{i}\left(\left\|b_{x, i}^{n+1, C}\right\|_{J}^{2}+\left\|b_{x, i+\frac{1}{2}}^{n+1, D}\right\|_{J}^{2}-\left\|b_{x, i}^{n, C}\right\|_{J}^{2}-\left\|b_{x, i+\frac{1}{2}}^{n, D}\right\|_{J}^{2}\right) \\
\leq & \left(-\frac{1}{2}+\alpha_{1}+\frac{\alpha_{2}\left(\left|u_{x}\right|+\left|u_{y}\right|\right) \tau}{h_{y}}\right) \sum_{i}\left\|B_{x, h}^{n+1, D}\left(x_{i+\frac{1}{2}}, \cdot\right)-B_{x, h}^{n, D}\left(x_{i+\frac{1}{2}}, \cdot\right)\right\|_{J}^{2} \\
& +\left(-\frac{1}{2}+\alpha_{1}+\frac{\alpha_{2}\left(\left|u_{x}\right|+\left|u_{y}\right|\right) \tau}{h_{y}}\right) \sum_{i}\left\|B_{x, h}^{n+1, C}\left(x_{i}, \cdot\right)-B_{x, h}^{n, C}\left(x_{i}, \cdot\right)\right\|_{J}^{2} \\
& +\left(-\frac{\theta}{2}+\frac{\theta^{2}}{4 \alpha_{1}}+\frac{\left|u_{y}\right| \tau}{\alpha_{2} h_{y}}\right) \sum_{i} \sum_{s=i \pm \frac{1}{2}}\left\|B_{x, h}^{n, D}\left(x_{s}, \cdot\right)-B_{x, h}^{n, C}\left(x_{i}, \cdot\right)\right\|_{J}^{2} \\
& +\frac{\left|u_{x}\right| \tau}{\alpha_{2} h_{x}} \sum_{j} \sum_{s=j \pm \frac{1}{2}}\left\|B_{y, h}^{n, D}\left(\cdot, y_{s}\right)-B_{y, h}^{n, C}\left(\cdot, y_{j}\right)\right\|_{I}^{2} .
\end{aligned}
$$

Similarly we can work with the second and fourth equations in (2.6) by interchanging the indices $x$ and $y, i$ and $j$, and get an estimate for $\frac{1}{2} \sum_{j}\left(\left\|b_{y, j}^{n+1, C}\right\|_{I}^{2}+\left\|b_{y, j+\frac{1}{2}}^{n+1, D}\right\|_{I}^{2}-\left\|b_{y, j}^{n, C}\right\|_{I}^{2}-\left\|b_{y, j+\frac{1}{2}}^{n, D}\right\|_{I}^{2}\right)$. Combining this estimate and (4.20), one gets for any $\alpha_{1}, \alpha_{2}>0$,

$$
\begin{aligned}
& \frac{1}{2}\left(\mathcal{E}_{h}\left(\mathbf{B}_{h}^{n+1, C}, \mathbf{B}_{h}^{n+1, D}\right)-\mathcal{E}_{h}\left(\mathbf{B}_{h}^{n, C}, \mathbf{B}_{h}^{n, D}\right)\right) \\
\leq & h_{x}\left(-\frac{1}{2}+\alpha_{1}+\frac{\alpha_{2}\left(\left|u_{x}\right|+\left|u_{y}\right|\right) \tau}{h_{y}}\right) \sum_{i}\left\|B_{x, h}^{n+1, D}\left(x_{i+\frac{1}{2}}, \cdot\right)-B_{x, h}^{n, D}\left(x_{i+\frac{1}{2}}, \cdot\right)\right\|_{J}^{2} \\
& +h_{x}\left(-\frac{1}{2}+\alpha_{1}+\frac{\alpha_{2}\left(\left|u_{x}\right|+\left|u_{y}\right|\right) \tau}{h_{y}}\right) \sum_{i}\left\|B_{x, h}^{n+1, C}\left(x_{i}, \cdot\right)-B_{x, h}^{n, C}\left(x_{i}, \cdot\right)\right\|_{J}^{2} \\
& +h_{y}\left(-\frac{1}{2}+\alpha_{1}+\frac{\alpha_{2}\left(\left|u_{x}\right|+\left|u_{y}\right|\right) \tau}{h_{x}}\right) \sum_{j}\left\|B_{y, h}^{n+1, D}\left(\cdot, y_{j+\frac{1}{2}}\right)-B_{y, h}^{n, D}\left(\cdot, y_{j+\frac{1}{2}}\right)\right\|_{I}^{2} \\
& +h_{y}\left(-\frac{1}{2}+\alpha_{1}+\frac{\alpha_{2}\left(\left|u_{x}\right|+\left|u_{y}\right|\right) \tau}{h_{x}}\right) \sum_{j}\left\|B_{y, h}^{n+1, C}\left(\cdot, y_{j}\right)-B_{y, h}^{n, C}\left(\cdot, y_{j}\right)\right\|_{I}^{2} \\
& +h_{x}\left(-\frac{\theta}{2}+\frac{\theta^{2}}{4 \alpha_{1}}+\frac{\left|u_{y}\right| \tau}{\alpha_{2} h_{y}}+\frac{\left|u_{y}\right| \tau}{\alpha_{2} h_{x}}\right) \sum_{i} \sum_{s=i \pm \frac{1}{2}}\left\|B_{x, h}^{n, D}\left(x_{s}, \cdot\right)-B_{x, h}^{n, C}\left(x_{i}, \cdot\right)\right\|_{J}^{2} \\
& +h_{y}\left(-\frac{\theta}{2}+\frac{\theta^{2}}{4 \alpha_{1}}+\frac{\left|u_{x}\right| \tau}{\alpha_{2} h_{x}}+\frac{\left|u_{x}\right| \tau}{\alpha_{2} h_{y}}\right) \sum_{j} \sum_{s=j \pm \frac{1}{2}}\left\|B_{y, h}^{n, D}\left(\cdot, y_{s}\right)-B_{y, h}^{n, C}\left(\cdot, y_{j}\right)\right\|_{I}^{2} .
\end{aligned}
$$


In order to achieve $\mathcal{E}_{h}\left(\mathbf{B}_{h}^{n+1, C}, \mathbf{B}_{h}^{n+1, D}\right) \leq \mathcal{E}_{h}\left(\mathbf{B}_{h}^{n, C}, \mathbf{B}_{h}^{n, D}\right)$, we require that the coefficients in front of all terms on the right-hand side of (4.21) are non-positive. That is,

$$
\begin{aligned}
-\frac{1}{2}+\alpha_{1}+\frac{\alpha_{2}\left(\left|u_{x}\right|+\left|u_{y}\right|\right) \tau}{h_{y}} \leq 0, & -\frac{1}{2}+\alpha_{1}+\frac{\alpha_{2}\left(\left|u_{x}\right|+\left|u_{y}\right|\right) \tau}{h_{x}} \leq 0, \\
-\frac{\theta}{2}+\frac{\theta^{2}}{4 \alpha_{1}}+\frac{\left|u_{y}\right| \tau}{\alpha_{2} h_{y}}+\frac{\left|u_{y}\right| \tau}{\alpha_{2} h_{x}} \leq 0, & -\frac{\theta}{2}+\frac{\theta^{2}}{4 \alpha_{1}}+\frac{\left|u_{x}\right| \tau}{\alpha_{2} h_{x}}+\frac{\left|u_{x}\right| \tau}{\alpha_{2} h_{y}} \leq 0 .
\end{aligned}
$$

This first puts a constraint on the constant $\alpha_{1}$, namely, $\frac{\theta}{2}<\alpha_{1}<\frac{1}{2}$. Under this condition, one can easily see that the four inequalities in (4.22) are equivalent to

$$
\tau \leq \min \left(\frac{\gamma\left(\alpha_{1}\right)}{\alpha_{2}}, \vartheta\left(\alpha_{1}\right) \alpha_{2}\right)
$$

where $\gamma\left(\alpha_{1}\right)=\left(\frac{1}{2}-\alpha_{1}\right) \frac{\min \left(h_{x}, h_{y}\right)}{\left|u_{x}\right|+\left|u_{y}\right|}$ and $\vartheta\left(\alpha_{1}\right)=\left(\frac{\theta}{2}-\frac{\theta^{2}}{4 \alpha_{1}}\right) \frac{h_{x} h_{y}}{\max \left(\left|u_{x}\right|,\left|u_{y}\right|\right)\left(h_{x}+h_{y}\right)}$. Finally we carefully choose $\alpha_{1} \in\left(\frac{\theta}{2}, \frac{1}{2}\right)$ and $\alpha_{2}>0$, so that the upper bound of (4.23) is maximized and hence provides the best condition for the time step $\tau$ for any fixed $\theta \in(0,1)$ to ensure the numerical stability.

$$
\begin{aligned}
\tau & \leq \max _{\alpha_{1} \in\left(\frac{\theta}{2}, \frac{1}{2}\right)} \max _{\alpha_{2}>0} \min \left(\frac{\gamma\left(\alpha_{1}\right)}{\alpha_{2}}, \vartheta\left(\alpha_{1}\right) \alpha_{2}\right) \\
& =\max _{\alpha_{1} \in\left(\frac{\theta}{2}, \frac{1}{2}\right)} \sqrt{\gamma\left(\alpha_{1}\right) \vartheta\left(\alpha_{1}\right)} \quad\left(\text { achieved at } \alpha_{2}=\sqrt{\gamma\left(\alpha_{1}\right) / \vartheta\left(\alpha_{1}\right)}\right) \\
& =\max _{\alpha_{1} \in\left(\frac{\theta}{2}, \frac{1}{2}\right)} \sqrt{\left(\frac{1}{2}-\alpha_{1}\right)\left(\frac{\theta}{2}-\frac{\theta^{2}}{4 \alpha_{1}}\right) \frac{\min \left(h_{x}, h_{y}\right) h_{x} h_{y}}{\max \left(\left|u_{x}\right|,\left|u_{y}\right|\right)\left(\left|u_{x}\right|+\left|u_{y}\right|\right)\left(h_{x}+h_{y}\right)}} \\
& =\sqrt{\theta}\left(\frac{1}{2}-\frac{\sqrt{\theta}}{2}\right) \sqrt{\frac{\min \left(h_{x}, h_{y}\right) h_{x} h_{y}}{\max \left(\left|u_{x}\right|,\left|u_{y}\right|\right)\left(\left|u_{x}\right|+\left|u_{y}\right|\right)\left(h_{x}+h_{y}\right)}} \quad\left(\text { achieved at } \alpha_{1}=\frac{\sqrt{\theta}}{2}\right) .
\end{aligned}
$$

Remark 4.5. Compared with the Fourier analysis, the CFL condition for stability via the energy analysis is much more pessimistic. In fact, this discrepancy can be even illustrated well when Fourier and energy analyses are applied to central DG methods solving the simple advection equation $u_{t}+a u_{x}=0$. What leads to this sub-optimal stability condition in energy analysis is the use of the Young's inequality to bound $\Lambda_{2}$ and $\Lambda_{4}$ in the proof of Theorem 4.1 in Section 4.1.2. A much improved CFL condition can be obtained if one works with $\Lambda_{i}, i=1, \ldots, 4$ all together and maximizes a quadratic function with a finite-dimensional input. The details are more tedious and hence not presented here.

\subsection{Error estimate}

In this section, we will establish the $L^{2}$ error estimate of the proposed method up to any given time $T>0$ when the exact solution has sufficient regularity. Without loss of generality, it is assumed $h<\frac{1}{2}$. The main result is stated in next Theorem.

Theorem 4.6. Let $\mathbf{B}$ be a sufficiently smooth exact solution to the magnetic induction equations (1.1), with the initial data being divergence-free, let $\mathbf{B}^{n}$ denote the exact solution $\mathbf{B}$ at $t^{n}$. With any given $\theta \in(0,1)$ in (2.6), let $\mathbf{B}_{h}^{n, \star} \in \mathcal{M}_{h}^{\star}$ be the numerical solution of the proposed scheme in (2.6)-(2.8) with the initialization $\mathbf{B}_{h}^{\star}=\Pi_{h}^{\star}\left(\left.\mathbf{B}\right|_{t=0}\right)$. As long as the time step $\tau$ satisfies $\tau=\sigma \tau_{\text {stab }}$ with $\sigma \leq 1$, we have

$$
\begin{aligned}
& \left\|\mathbf{B}^{n}-\mathbf{B}_{h}^{n, C}\right\|_{0, \Omega}^{2}+\left\|\mathbf{B}^{n}-\mathbf{B}_{h}^{n, D}\right\|_{0, \Omega}^{2} \\
& \leq C^{\dagger}\left(\exp \left(T \frac{h_{x}+h_{y}}{\tau}\right)\left\{\theta^{2} \frac{\left(h_{x}+h_{y}\right)^{5}}{h_{x} h_{y}}+\left(\left|u_{x}\right|+\left|u_{y}\right|\right)^{2} \frac{\tau^{2}\left(h_{x}+h_{y}\right)^{5}}{h_{x} h_{y}}+\frac{\tau^{4}}{h_{x} h_{y}}\right\}+h^{2}\right),
\end{aligned}
$$


for any $n: n \tau \leq T$. Here the positive constant $C^{\dagger}$ depends on some Sobolev norms of the exact solution on $\Omega \times(0, T),|\Omega|,|I|,|J|$, and $T$, and it is independent of $\tau, h$ and $n$. Moreover, if we further require

$$
\delta_{1} h_{x} \leq h_{y} \leq \delta_{2} h_{x}
$$

for some positive constant $\delta_{1}, \delta_{2}$ independent of $h_{x}, h_{y}$ during mesh refinements, then

$$
\left\|\mathbf{B}^{n}-\mathbf{B}_{h}^{n, C}\right\|_{0, \Omega}^{2}+\left\|\mathbf{B}^{n}-\mathbf{B}_{h}^{n, D}\right\|_{0, \Omega}^{2} \leq C^{\dagger} C_{\dagger}\left(\tau^{2}+h^{2}\right) .
$$

Here the constant $C_{\dagger}$ depends on $\sigma, \delta_{1}, \delta_{2}, u_{x}, u_{y}$, and $\theta$.

From this Theorem, one can see that the exactly divergence-free method described in Section 2.2 is of first order accuracy in both space and time, and this is somewhat expected. We want to mention that central DG methods in general can be only proved to be $k$ th order accurate when piecewise polynomials of degree at most $k$ are used as discrete spaces to solve the linear advection equation [16].

To make the proof easier to follow, we will organize the following subsections just as in Section 4.1: in Section 4.2.1 we state some preparatory lemmas, based on which the proof of the main error estimate (4.24) will be established in Section 4.2.2. Notation wise, we define

$$
\eta^{n, \star}=\left[\eta_{x}^{n, \star}, \eta_{y}^{n, \star}\right]^{\top}=\mathbf{B}^{n}-\Pi_{h}^{\star} \mathbf{B}^{n}, \quad \xi^{n, \star}=\left[\xi_{x}^{n, \star}, \xi_{y}^{n, \star}\right]^{\top}=\mathbf{B}_{h}^{n, \star}-\Pi_{h}^{\star} \mathbf{B}^{n},
$$

that is, the error in numerical solutions $\mathbf{B}^{n}-\mathbf{B}_{h}^{n, \star}$ is decomposed into

$$
\mathbf{B}^{n}-\mathbf{B}_{h}^{n, \star}=\eta^{n, \star}-\xi^{n, \star} .
$$

In particular, $\xi^{n, \star}=-\Pi_{h}^{\star}\left(\mathbf{B}^{n}-\mathbf{B}_{h}^{n, \star}\right)$ corresponds to (the negative of) the projection of the numerical error. Throughout our analysis, the constant $C^{\dagger}$ may take different values at different occurrences. It may depend partially on some Sobolev norms of the exact solution on $\Omega \times(0, T),|\Omega|,|I|,|J|$, and $T$, yet it is independent of $\tau, h$ and $n$. For simplicity of the presentation, the regularity of the exact solution is measured by $L^{\infty}$ type norm, namely $\|\cdot\|_{W^{m, \infty}(\Omega)}$. This can be relaxed to $L^{2}$ type norm, namely $\|\cdot\|_{H^{m}(\Omega)}$, if Taylor expansion with Lagrange remainder is used instead for analysis.

Remark 4.7. The error estimates in this section will be established by following energy approach. This framework involves quite much analysis, yet it can lead to optimal error estimates for "hybrid" numerical methods, such as the one considered here and the related high-order ones [13], which involve finite element type spatial discretizations and explicit Runge-Kutta time discretizations. If one instead analyzes such hybrid methods, especially those with higher order accuracy, by treating them as finite difference methods and combing stability and local truncation errors, one may encounter the supraconvergence phenomenon and only gets sub-optimal error estimates (see [23] and the references therein). If it were not to prepare for understanding and analyzing the "hybrid" divergence-free schemes of higher order accuracy, one can alternatively carry out a simpler error estimate for the first order scheme considered in this paper.

\subsubsection{Preliminary lemmas}

Among the following lemmas, Lemma 4.8 describes some orthogonality property related to the projection operators. Lemmas 4.9 and 4.10 reflect the approximation property of the discrete spaces. Although the proofs of Lemmas 4.9 and 4.10 seem to only use basic ingredients such as Taylor expansion, we want to point out that a simple application of some standard inequality, such as $\|\phi \cdot n\|_{\partial K}^{2} \leq \mathcal{C}\left(\frac{1}{\min \left(h_{x}, h_{y}\right)}\|\phi\|_{K}^{2}+\|\nabla \cdot \phi\|_{K}^{2}\right), \forall \phi \in$ $H(\operatorname{div} ; \Omega)$, will not be sufficient in order to estimate the projection errors in these lemmas. Lemma 4.11 is to bound the local truncation errors in time.

Lemma 4.8. Let $\phi=\left[\phi_{x}, \phi_{y}\right]^{\top} \in \mathcal{M}_{h}^{D}, \varphi=\left[\varphi_{x}, \varphi_{y}\right]^{\top} \in \mathcal{M}_{h}^{C}$, then $\forall i, j, n$,

$$
\begin{gathered}
\left(\eta_{x}^{n, C}\left(x_{i}, \cdot\right), \varphi_{x}\left(x_{i}, \cdot\right)\right)_{J_{j+\frac{1}{2}}}=0, \quad\left(\eta_{y}^{n, C}\left(\cdot, y_{j}\right), \varphi_{y}\left(\cdot, y_{j}\right)\right)_{I_{i+\frac{1}{2}}}=0, \\
\left(\eta_{x}^{n, D}\left(x_{i+\frac{1}{2}}, \cdot\right), \phi_{x}\left(x_{i+\frac{1}{2}}, \cdot\right)\right)_{J_{j}}=0, \quad\left(\eta_{y}^{n, D}\left(\cdot, y_{j+\frac{1}{2}}\right), \phi_{y}\left(\cdot, y_{j+\frac{1}{2}}\right)\right)_{I_{i}}=0 .
\end{gathered}
$$


The orthogonality results in this lemma can be directly obtained from the definition of the projection operator $\Pi_{h}^{\star}$ in $(2.3)$ and the property of $\mathcal{W}(K)$ in $(2.2 \mathrm{a})$.

Lemma 4.9. Let $\phi=\left[\phi_{x}, \phi_{y}\right]^{\top} \in \mathcal{M}_{h}^{D}, \varphi=\left[\varphi_{x}, \varphi_{y}\right]^{\top} \in \mathcal{M}_{h}^{C}$, then $\forall \alpha>0$,

$$
\begin{gathered}
\sum_{i}\left(\eta_{x}^{n, D}\left(x_{i}, \cdot\right)-\eta_{x}^{n, C}\left(x_{i}, \cdot\right), \varphi_{x}\left(x_{i}, \cdot\right)\right)_{J} \leq \frac{C^{\dagger}}{\alpha} \frac{\left(h_{x}+h_{y}\right)^{4} h_{y}}{h_{x}^{2}}+\alpha h_{x} \sum_{i}\left\|\varphi_{x}\left(x_{i}, \cdot\right)\right\|_{J}^{2}, \\
\sum_{i}\left(\eta_{x}^{n, C}\left(x_{i+\frac{1}{2}}, \cdot\right)-\eta_{x}^{n, D}\left(x_{i+\frac{1}{2}}, \cdot\right), \phi_{x}\left(x_{i+\frac{1}{2}}, \cdot\right)\right)_{J} \leq \frac{C^{\dagger}}{\alpha} \frac{\left(h_{x}+h_{y}\right)^{4} h_{y}}{h_{x}^{2}}+\alpha h_{x} \sum_{i}\left\|\phi_{x}\left(x_{i+\frac{1}{2}}, \cdot\right)\right\|_{J}^{2} .
\end{gathered}
$$

Proof. With similarity, we here only prove (4.30a). Based on the explicit formulation of the projection operator $\Pi_{h}^{D}$ in (2.4) and applying Taylor expansion, we have

$$
\begin{aligned}
& \int_{y_{j}}^{y_{j+1}} \eta_{x}^{n, D}\left(x_{i}, y\right) \mathrm{d} y=\int_{y_{j}}^{y_{j+\frac{1}{2}}} \eta_{x}^{n, D}\left(x_{i}, y\right) \mathrm{d} y+\int_{y_{j+\frac{1}{2}}}^{y_{j+1}} \eta_{x}^{n, D}\left(x_{i}, y\right) \mathrm{d} y \\
= & \int_{y_{j}}^{y_{j+1}} B_{x}^{n}\left(x_{i}, s\right)-\frac{1}{4}\left(B_{x}^{n}\left(x_{i-\frac{1}{2}}, s-\frac{h_{y}}{2}\right)+B_{x}^{n}\left(x_{i+\frac{1}{2}}, s-\frac{h_{y}}{2}\right)\right. \\
& \left.+B_{x}^{n}\left(x_{i-\frac{1}{2}}, s+\frac{h_{y}}{2}\right)+B_{x}^{n}\left(x_{i+\frac{1}{2}}, s+\frac{h_{y}}{2}\right)\right) \mathrm{d} s \\
\leq & C^{\dagger}\left(h_{x}+h_{y}\right)^{2} h_{y},
\end{aligned}
$$

where the positive constant $C^{\dagger}$ depends on the $L^{\infty}$ norm of the second spatial derivatives of the exact solution $B_{x}$. With this, we next use the orthogonality result in Lemma 4.8 and the property of $\mathcal{W}(K)$ in $(2.2 \mathrm{a})$, and get

$$
\begin{aligned}
& \int_{y_{j}}^{y_{j+1}}\left(\eta_{x}^{n, D}\left(x_{i}, y\right)-\eta_{x}^{n, C}\left(x_{i}, y\right)\right) \varphi_{x}\left(x_{i}, y\right) \mathrm{d} y \\
= & \int_{y_{j}}^{y_{j+1}} \eta_{x}^{n, D}\left(x_{i}, y\right) \varphi_{x}\left(x_{i}, y\right) \mathrm{d} y=\varphi_{x}\left(x_{i}, y_{j+\frac{1}{2}}\right) \int_{y_{j}}^{y_{j+1}} \eta_{x}^{n, D}\left(x_{i}, y\right) \mathrm{d} y \\
\leq & C^{\dagger} \sum_{i, j}\left(h_{x}+h_{y}\right)^{2} h_{y}^{\frac{1}{2}}\left\|\varphi_{x}\left(x_{i}, \cdot\right)\right\|_{J_{j+\frac{1}{2}}}=C^{\dagger} \sum_{i}\left(h_{x}+h_{y}\right)^{2} h_{y}^{\frac{1}{2}}\left\|\varphi_{x}\left(x_{i}, \cdot\right)\right\|_{J} \\
\leq & \frac{C^{\dagger}}{\alpha} \sum_{i} \frac{\left(h_{x}+h_{y}\right)^{4} h_{y}}{h_{x}}+\alpha h_{x} \sum_{i}\left\|\varphi_{x}\left(x_{i}, \cdot\right)\right\|_{J}^{2}=\frac{C^{\dagger}}{\alpha} \frac{\left(h_{x}+h_{y}\right)^{4} h_{y}}{h_{x}^{2}}+\alpha h_{x} \sum_{i}\left\|\varphi_{x}\left(x_{i}, \cdot\right)\right\|_{J}^{2} .
\end{aligned}
$$

Here the constant $C^{\dagger}$ also depends on $I$. This concludes (4.30a).

Lemma 4.10. Let $\phi=\left[\phi_{x}, \phi_{y}\right]^{\top} \in \mathcal{M}_{h}^{D}, \varphi=\left[\varphi_{x}, \varphi_{y}\right]^{\top} \in \mathcal{M}_{h}^{C}$, then $\forall \alpha>0$,

$$
\begin{aligned}
& \text { (i) } \sum_{i, j} G_{j+\frac{1}{2}}\left(\eta_{x}^{n, D}\left(x_{i}, \cdot\right), \varphi_{x}\left(x_{i}, \cdot\right)\right) \leq \frac{C^{\dagger}}{\alpha} \frac{\left(h_{x}+h_{y}\right)^{6}}{h_{x}^{2} h_{y}}+\alpha h_{x} \sum_{i}\left\|\varphi_{x}\left(x_{i}, \cdot\right)\right\|_{J}^{2}, \\
& \text { (ii) } \sum_{i, j} G_{j+\frac{1}{2}}\left(\eta_{y}^{n, D}\left(x_{i}, \cdot\right), \varphi_{x}\left(x_{i}, \cdot\right)\right) \leq \frac{C^{\dagger}}{\alpha} \frac{\left(h_{x}+h_{y}\right)^{6}}{h_{x}^{2} h_{y}}+\alpha h_{x} \sum_{i}\left\|\varphi_{x}\left(x_{i}, \cdot\right)\right\|_{J}^{2} .
\end{aligned}
$$

Similarly, both $\sum_{i, j} G_{j}\left(\eta_{x}^{n, C}\left(x_{i+\frac{1}{2}}, \cdot\right), \phi_{x}\left(x_{i+\frac{1}{2}}, \cdot\right)\right)$ and $\sum_{i, j} G_{j}\left(\eta_{y}^{n, C}\left(x_{i+\frac{1}{2}}, \cdot\right), \phi_{x}\left(x_{i+\frac{1}{2}}, \cdot\right)\right)$ can be bounded by $\frac{C^{\dagger}}{\alpha} \frac{\left(h_{x}+h_{y}\right)^{6}}{h_{x}^{2} h_{y}}+\alpha h_{x} \sum_{i}\left\|\phi_{x}\left(x_{i+\frac{1}{2}}, \cdot\right)\right\|_{J}^{2}$. 
Proof. We start with part (i). Based on the explicit formulation of the projection operator $\Pi_{h}^{D}$ in (2.4) and applying Taylor expansion, we have

$$
\begin{aligned}
& \eta_{x}^{n, D}\left(x_{i}, y_{j+1}\right)-\eta_{x}^{n, D}\left(x_{i}, y_{j}\right) \\
= & B_{x}^{n}\left(x_{i}, y_{j+1}\right)-B_{x}^{n}\left(x_{i}, y_{j}\right)-\frac{1}{2 h_{y}} \int_{y_{j-\frac{1}{2}}}^{y_{j+\frac{1}{2}}}\left(B_{x}^{n}\left(x_{i-\frac{1}{2}}, s+h_{y}\right)-B_{x}^{n}\left(x_{i-\frac{1}{2}}, s\right)\right) \mathrm{d} s \\
& -\frac{1}{2 h_{y}} \int_{y_{j-\frac{1}{2}}}^{y_{j+\frac{1}{2}}}\left(B_{x}^{n}\left(x_{i+\frac{1}{2}}, s+h_{y}\right)-B_{x}^{n}\left(x_{i+\frac{1}{2}}, s\right)\right) \mathrm{d} s \\
= & B_{x}^{n}\left(x_{i}, y_{j+1}\right)-B_{x}^{n}\left(x_{i}, y_{j}\right)-\frac{1}{2 h_{y}} \int_{y_{j-\frac{1}{2}}}^{y_{j+\frac{1}{2}}} 2 h_{y} \frac{\partial B_{x}^{n}}{\partial y}\left(x_{i}, s\right) \\
& +h_{y}^{2} \frac{\partial^{2} B_{x}^{n}}{\partial y^{2}}\left(x_{i}, s\right) \mathrm{d} s+C^{\dagger}\left(h_{x}^{3}+h_{x}^{2} h_{y}+h_{x} h_{y}^{2}+h_{y}^{3}\right),
\end{aligned}
$$

hence

$$
\left|\eta_{x}^{n, D}\left(x_{i}, y_{j+1}\right)-\eta_{x}^{n, D}\left(x_{i}, y_{j}\right)\right| \leq C^{\dagger}\left(h_{x}+h_{y}\right)^{3} .
$$

Here the positive constant $C^{\dagger}$ depends on the $L^{\infty}$ norm of the third derivatives of the exact solution $B_{x}$.

With (4.35) and $\left.\varphi_{x}\left(x_{i}, y\right)\right|_{J_{j+\frac{1}{2}}}$ being constant, we obtain (4.34a) with $\alpha>0$,

$$
\begin{aligned}
& \sum_{i, j} G_{j+\frac{1}{2}}\left(\eta_{x}^{n, D}\left(x_{i}, y\right), \varphi_{x}\left(x_{i}, y\right)\right)=-\sum_{i, j}\left(\eta_{x}^{n, D}\left(x_{i}, y_{j+1}\right)-\eta_{x}^{n, D}\left(x_{i}, y_{j}\right)\right) \varphi_{x}\left(x_{i}, y_{j+\frac{1}{2}}\right) \\
\leq & \sum_{i, j} C^{\dagger}\left(h_{x}+h_{y}\right)^{3} h_{y}^{-\frac{1}{2}}\left\|\varphi_{x}\left(x_{i}, \cdot\right)\right\|_{J_{j+\frac{1}{2}}}=\sum_{i} C^{\dagger}\left(h_{x}+h_{y}\right)^{3} h_{y}^{-\frac{1}{2}}\left\|\varphi_{x}\left(x_{i}, \cdot\right)\right\|_{J} \\
\leq & \frac{C^{\dagger}}{\alpha} \frac{\left(h_{x}+h_{y}\right)^{6}}{h_{x}^{2} h_{y}}+\alpha h_{x} \sum_{i}\left\|\varphi_{x}\left(x_{i}, \cdot\right)\right\|_{J}^{2} .
\end{aligned}
$$

Next we prove part (ii). Based on the explicit formulation of the projection operator $\Pi_{h}^{D}$ in (2.4), Taylor expansion, and using the exact solution being divergence-free, we have

$$
\begin{aligned}
& \eta_{y}^{n, D}\left(x_{i}, y_{j+1}\right)-\eta_{y}^{n, D}\left(x_{i}, y_{j}\right) \\
= & B_{y}^{n}\left(x_{i}, y_{j+1}\right)-B_{y}^{n}\left(x_{i}, y_{j}\right)-\frac{1}{2 h_{x}} \int_{x_{i-\frac{1}{2}}}^{x_{i+\frac{1}{2}}}\left(B_{y}^{n}\left(x, y_{j+\frac{3}{2}}\right)-B_{y}^{n}\left(x, y_{j-\frac{1}{2}}\right)\right) \mathrm{d} x \\
= & h_{y}\left(\frac{\partial B_{y}^{n}}{\partial y}\left(x_{i}, y_{j+\frac{1}{2}}\right)-\frac{1}{h_{x}} \int_{x_{i-\frac{1}{2}}}^{x_{i+\frac{1}{2}}} \frac{\partial B_{y}^{n}}{\partial y}\left(x, y_{j+\frac{1}{2}}\right) \mathrm{d} x\right)+C^{\dagger} h_{y}^{3} \\
= & -h_{y}\left(\frac{\partial B_{x}^{n}}{\partial x}\left(x_{i}, y_{j+\frac{1}{2}}\right)-\frac{1}{h_{x}}\left(B_{x}^{n}\left(x_{i+\frac{1}{2}}, y_{j+\frac{1}{2}}\right)-B_{x}^{n}\left(x_{i-\frac{1}{2}}, y_{j+\frac{1}{2}}\right)\right)\right)+C^{\dagger} h_{y}^{3} \leq C^{\dagger}\left(h_{x}+h_{y}\right)^{3} .
\end{aligned}
$$

With this and a similar argument for part (i), we will obtain the estimate in (4.34b).

Although the exact solution being divergence-free is used in the proof of Lemma 4.10, this is not essential for the error estimate.

Lemma 4.11. Let $T_{B, x}^{n}(x, y)=B_{x}^{n+1}(x, y)-B_{x}^{n}(x, y)-\tau \frac{\partial B_{x}^{n}(x, y)}{\partial t}$ be the local truncation error in time, and define $T_{B, x, i}^{n}(y)=T_{B, x}^{n}\left(x_{i}, y\right), T_{B, x, i+\frac{1}{2}}^{n}(y)=T_{B, x}^{n}\left(x_{i+\frac{1}{2}}, y\right)$, and let $\phi=\left[\phi_{x}, \phi_{y}\right]^{\top} \in \mathcal{M}_{h}^{D}, \varphi=\left[\varphi_{x}, \varphi_{y}\right]^{\top} \in \mathcal{M}_{h}^{C}$, 
then $\forall \alpha>0$,

$$
\begin{gathered}
\sum_{i}\left(T_{B, x, i}^{n}(\cdot), \varphi_{x}\left(x_{i}, \cdot\right)\right)_{J} \leq \frac{C^{\dagger}}{\alpha} \frac{\tau^{4}}{h_{x}^{2}}+\alpha h_{x} \sum_{i}\left\|\varphi_{x}\left(x_{i}, \cdot\right)\right\|_{J}^{2}, \\
\sum_{i}\left(T_{B, x, i+\frac{1}{2}}^{n}(\cdot), \phi_{x}\left(x_{i+\frac{1}{2}}, \cdot\right)\right)_{J} \leq \frac{C^{\dagger}}{\alpha} \frac{\tau^{4}}{h_{x}^{2}}+\alpha h_{x} \sum_{i}\left\|\phi_{x}\left(x_{i+\frac{1}{2}}, \cdot\right)\right\|_{J}^{2}
\end{gathered}
$$

The estimates in Lemma 4.11 are direct results of Taylor expansion, and the proofs are omitted.

\subsubsection{Error estimates: proof of Theorem 4.6}

Our error analysis starts from the error equations, which essentially are the equations satisfied by the errors in numerical solutions. Based on these equations, we will derive the energy equations, which measure the increase of the projection of the errors over one time step, and will be bounded by using the preparatory lemmas in Sections 4.1.1 and 4.2.1. In the end, the overall error up to a given time $T$ is estimated based on its projection and the approximation properties of the discrete spaces.

\subsubsection{Error equations and energy equations}

Based on the proposed method (2.6)-(2.8), the governing equations (1.2), and the decomposition of the errors in (4.28), one can easily get one of the error equations, with any $\left.\mu_{1}(y)\right|_{J_{j+\frac{1}{2}}} \in \mathbb{R}$,

$$
\begin{aligned}
& \left(\xi_{x}^{n+1, C}\left(x_{i}, \cdot\right)-\xi_{x}^{n, C}\left(x_{i}, \cdot\right), \mu_{1}(\cdot)\right)_{J_{j+\frac{1}{2}}} \\
= & \theta\left(\xi_{x}^{n, D}\left(x_{i}, \cdot\right)-\xi_{x}^{n, C}\left(x_{i}, \cdot\right), \mu_{1}(\cdot)\right)_{J_{j+\frac{1}{2}}}+\tau G_{j+\frac{1}{2}}\left(u_{y} \xi_{x}^{n, D}\left(x_{i}, \cdot\right)-u_{x} \xi_{y}^{n, D}\left(x_{i}, \cdot\right), \mu_{1}(\cdot)\right) \\
& +\left(\eta_{x}^{n+1, C}\left(x_{i}, \cdot\right)-\eta_{x}^{n, C}\left(x_{i}, \cdot\right), \mu_{1}(\cdot)\right)_{J_{j+\frac{1}{2}}}-\theta\left(\eta_{x}^{n, D}\left(x_{i}, \cdot\right)-\eta_{x}^{n, C}\left(x_{i}, \cdot\right), \mu_{1}(\cdot)\right)_{J_{j+\frac{1}{2}}} \\
& -\tau G_{j+\frac{1}{2}}\left(u_{y} \eta_{x}^{n, D}\left(x_{i}, \cdot\right)-u_{x} \eta_{y}^{n, D}\left(x_{i}, \cdot\right), \mu_{1}(\cdot)\right)-\left(T_{B, x, i}(\cdot), \mu_{1}(\cdot)\right)_{J_{j+\frac{1}{2}}} .
\end{aligned}
$$

By formally shifting the index $i$ to $i+\frac{1}{2}, j+\frac{1}{2}$ to $j, \mu_{1}$ to $\mu_{2}, C$ to $D$, we get one more error equation,

$$
\begin{aligned}
& \left(\xi_{x}^{n+1, D}\left(x_{i+\frac{1}{2}}, \cdot\right)-\xi_{x}^{n, D}\left(x_{i+\frac{1}{2}}, \cdot\right), \mu_{2}(\cdot)\right)_{J_{j}} \\
= & \theta\left(\xi_{x}^{n, C}\left(x_{i+\frac{1}{2}}, \cdot\right)-\xi_{x}^{n, D}\left(x_{i+\frac{1}{2}}, \cdot\right), \mu_{2}(\cdot)\right)_{J_{j}}+\tau G_{j}\left(u_{y} \xi_{x}^{n, C}\left(x_{i+\frac{1}{2}}, \cdot\right)-u_{x} \xi_{y}^{n, C}\left(x_{i+\frac{1}{2}}, \cdot\right), \mu_{2}(\cdot)\right) \\
& +\left(\eta_{x}^{n+1, D}\left(x_{i+\frac{1}{2}}, \cdot\right)-\eta_{x}^{n, D}\left(x_{i+\frac{1}{2}}, \cdot\right), \mu_{2}(\cdot)\right)_{J_{j}}-\theta\left(\eta_{x}^{n, C}\left(x_{i+\frac{1}{2}}, \cdot\right)-\eta_{x}^{n, D}\left(x_{i+\frac{1}{2}}, \cdot\right), \mu_{2}(\cdot)\right)_{J_{j}} \\
& -\tau G_{j}\left(u_{y} \eta_{x}^{n, C}\left(x_{i+\frac{1}{2}}, \cdot\right)-u_{x} \eta_{y}^{n, C}\left(x_{i+\frac{1}{2}}, \cdot\right), \mu_{2}(\cdot)\right)-\left(T_{B, x, i+\frac{1}{2}}(\cdot), \mu_{2}(\cdot)\right)_{J_{j}},
\end{aligned}
$$

with any $\left.\mu_{2}(y)\right|_{J_{j}} \in \mathbb{R}$. In particular, we take $\mu_{1}(y)=\xi_{x}^{n+1, C}\left(x_{i}, y\right)$ in $(4.38), \mu_{2}(y)=\xi_{x}^{n+1, D}\left(x_{i+\frac{1}{2}}, y\right)$ in (4.39), sum up in $i, j$, and get the part of the energy equations associated to the $x$-component of the numerical solution,

$$
\begin{aligned}
\frac{1}{2} \sum_{i}\left(\left\|\xi_{x}^{n+1, C}\left(x_{i}, \cdot\right)\right\|_{J}^{2}+\left\|\xi_{x}^{n+1, D}\left(x_{i+\frac{1}{2}}, \cdot\right)\right\|_{J}^{2}-\left\|\xi_{x}^{n, C}\left(x_{i}, \cdot\right)\right\|_{J}^{2}-\left\|\xi_{x}^{n, D}\left(x_{i+\frac{1}{2}}, \cdot\right)\right\|_{J}^{2}\right) \\
=-\frac{1}{2} \sum_{i}\left\|\xi_{x}^{n+1, C}\left(x_{i}, \cdot\right)-\xi_{x}^{n, C}\left(x_{i}, \cdot\right)\right\|_{J}^{2}-\frac{1}{2} \sum_{i}\left\|\xi_{x}^{n+1, D}\left(x_{i+\frac{1}{2}}, \cdot\right)-\xi_{x}^{n, D}\left(x_{i+\frac{1}{2}}, \cdot\right)\right\|_{J}^{2}+\sum_{i=1}^{5} \Theta_{i}
\end{aligned}
$$


where

$$
\begin{aligned}
& \Theta_{1}=\theta \sum_{i}\left(\left(\xi_{x}^{n, D}\left(x_{i}, \cdot\right)-\xi_{x}^{n, C}\left(x_{i}, \cdot\right), \xi_{x}^{n+1, C}\left(x_{i}, \cdot\right)\right)_{J}+\left(\xi_{x}^{n, C}\left(x_{i+\frac{1}{2}}, \cdot\right)-\xi_{x}^{n, D}\left(x_{i+\frac{1}{2}}, \cdot\right), \xi_{x}^{n+1, D}\left(x_{i+\frac{1}{2}}, \cdot\right)\right)_{J}\right) \\
& +\tau \sum_{i, j} G_{j+\frac{1}{2}}\left(u_{y} \xi_{x}^{n, D}\left(x_{i}, \cdot\right)-u_{x} \xi_{y}^{n, D}\left(x_{i}, \cdot\right), \xi_{x}^{n+1, C}\left(x_{i}, \cdot\right)\right) \\
& +\tau \sum_{i, j} G_{j}\left(u_{y} \xi_{x}^{n, C}\left(x_{i+\frac{1}{2}}, \cdot\right)-u_{x} \xi_{y}^{n, C}\left(x_{i+\frac{1}{2}}, \cdot\right), \xi_{x}^{n+1, D}\left(x_{i+\frac{1}{2}}, \cdot\right)\right), \\
& \Theta_{2}=\sum_{i}\left(\eta_{x}^{n+1, C}\left(x_{i}, \cdot\right)-\eta_{x}^{n, C}\left(x_{i}, \cdot\right), \xi_{x}^{n+1, C}\left(x_{i}, \cdot\right)\right)_{J}+\sum_{i}\left(\eta_{x}^{n+1, D}\left(x_{i+\frac{1}{2}}, \cdot\right)-\eta_{x}^{n, D}\left(x_{i+\frac{1}{2}}, \cdot\right), \xi_{x}^{n+1, D}\left(x_{i+\frac{1}{2}}, \cdot\right)\right)_{J}, \\
& \Theta_{3}=-\theta \sum_{i}\left(\left(\eta_{x}^{n, D}\left(x_{i}, \cdot\right)-\eta_{x}^{n, C}\left(x_{i}, \cdot\right), \xi_{x}^{n+1, C}\left(x_{i}, \cdot\right)\right)_{J}+\left(\eta_{x}^{n, C}\left(x_{i+\frac{1}{2}}, \cdot\right)-\eta_{x}^{n, D}\left(x_{i+\frac{1}{2}}, \cdot\right), \xi_{x}^{n+1, D}\left(x_{i+\frac{1}{2}}, \cdot\right)\right)_{J}\right), \\
& \Theta_{4}=-\tau \sum_{i, j} G_{j+\frac{1}{2}}\left(u_{y} \eta_{x}^{n, D}\left(x_{i}, \cdot\right)-u_{x} \eta_{y}^{n, D}\left(x_{i}, \cdot\right), \xi_{x}^{n+1, C}\left(x_{i}, \cdot\right)\right) \\
& -\tau \sum_{i, j} G_{j}\left(u_{y} \eta_{x}^{n, C}\left(x_{i+\frac{1}{2}}, \cdot\right)-u_{x} \eta_{y}^{n, C}\left(x_{i+\frac{1}{2}}, \cdot\right), \xi_{x}^{n+1, D}\left(x_{i+\frac{1}{2}}, \cdot\right)\right), \\
& \Theta_{5}=-\sum_{i}\left(T_{B, x, i}^{n}(\cdot), \xi_{x}^{n+1, C}\left(x_{i}, \cdot\right)\right)_{J}-\sum_{i}\left(T_{B, x, i+\frac{1}{2}}^{n}(\cdot), \xi_{x}^{n+1, D}\left(x_{i+\frac{1}{2}}, \cdot\right)\right)_{J} .
\end{aligned}
$$

One more part of the energy equations is related to $\xi_{y}^{n, \star}, \star=C, D$, it can be formally obtained by switching $i$ to $j, x$ to $y, J$ to $I$, and will not be given explicitly here to save space. Note that the energy equation (4.40) measures the increase of energy associated to the projected errors over one time step. Estimating such increase will be a key component in error estimates, and this will be discussed next.

\subsubsection{Estimation of energy equations}

To estimate $\Theta_{1}$ in (4.41), the same techniques in Section 4.1.2 to estimate $\Lambda_{1}-\Lambda_{4}$ for numerical stability will be applied, and this leads to

$$
\begin{aligned}
\Theta_{1} \leq & -\frac{\theta}{2} \sum_{i} \sum_{s=i \pm \frac{1}{2}}\left\|\xi_{x}^{n, D}\left(x_{s}, \cdot\right)-\xi_{x}^{n, C}\left(x_{i}, \cdot\right)\right\|_{J}^{2}+\frac{\theta^{2}}{4 \alpha_{1}} \sum_{i} \sum_{s=i \pm \frac{1}{2}}\left\|\xi_{x}^{n, D}\left(x_{s}, \cdot\right)-\xi_{x}^{n, C}\left(x_{i}, \cdot\right)\right\|_{J}^{2} \\
& +\alpha_{1} \sum_{i}\left\{\left\|\xi_{x}^{n+1, C}\left(x_{i}, \cdot\right)-\xi_{x}^{n, C}\left(x_{i}, \cdot\right)\right\|_{J}^{2}+\left\|\xi_{x}^{n+1, D}\left(x_{i+\frac{1}{2}}, \cdot\right)-\xi_{x}^{n, D}\left(x_{i+\frac{1}{2}}, \cdot\right)\right\|_{J}^{2}\right\} \\
& +\frac{\tau\left|u_{y}\right|}{\alpha_{2} h_{y}} \sum_{i} \sum_{s=i \pm \frac{1}{2}}\left\|\xi_{x}^{n, D}\left(x_{s}, \cdot\right)-\xi_{x}^{n, C}\left(x_{i}, \cdot\right)\right\|_{J}^{2}+\frac{\tau\left|u_{x}\right|}{\alpha_{2} h_{x}} \sum_{j} \sum_{s=j \pm \frac{1}{2}}\left\|\xi_{y}^{n, D}\left(\cdot, y_{s}\right)-\xi_{y}^{n, C}\left(\cdot, y_{j}\right)\right\|_{I}^{2} \\
& +\frac{\alpha_{2} \tau\left(\left|u_{x}\right|+\left|u_{y}\right|\right)}{h_{y}} \sum_{i}\left(\left\|\xi_{x}^{n+1, C}\left(x_{i}, \cdot\right)-\xi_{x}^{n, C}\left(x_{i}, \cdot\right)\right\|_{J}^{2}+\left\|\xi_{x}^{n+1, D}\left(x_{i+\frac{1}{2}}, \cdot\right)-\xi_{x}^{n, D}\left(x_{i+\frac{1}{2}}, \cdot\right)\right\|_{J}^{2}\right),
\end{aligned}
$$

with any $\alpha_{1}, \alpha_{2}>0$. 
Next we will turn to terms $\Theta_{i}, i=2, \ldots, 5$ in (4.42)-(4.45), which involve both $\xi_{\diamond}^{m, \star}$ and $\eta_{\diamond}^{m, \star}$, with $\diamond=x, y$, $\star=C, D$ and some integer $m$, and will be estimated based on Lemmas 4.8-4.11. In particular, from Lemma 4.8, we see $\Theta_{2}$ vanishes. The terms $\Theta_{3}, \Theta_{4}, \Theta_{5}$ will be estimated using Lemmas 4.9-4.11, respectively,

$$
\begin{aligned}
& \Theta_{3} \leq \theta^{2} \frac{C^{\dagger}}{\alpha_{3}} \frac{\left(h_{x}+h_{y}\right)^{4} h_{y}}{h_{x}^{2}}+\alpha_{3} h_{x} \sum_{i}\left(\left\|\xi_{x}^{n+1, C}\left(x_{i}, \cdot\right)\right\|_{J}^{2}+\left\|\xi_{x}^{n+1, D}\left(x_{i+\frac{1}{2}}, \cdot\right)\right\|_{J}^{2}\right), \\
& \Theta_{4} \leq\left(\left|u_{x}\right|+\left|u_{y}\right|\right)^{2} \frac{C^{\dagger}}{\alpha_{4}} \frac{\tau^{2}\left(h_{x}+h_{y}\right)^{6}}{h_{x}^{2} h_{y}}+\alpha_{4} h_{x} \sum_{i}\left(\left\|\xi_{x}^{n+1, C}\left(x_{i}, \cdot\right)\right\|_{J}^{2}+\left\|\xi_{x}^{n+1, D}\left(x_{i+\frac{1}{2}}, \cdot\right)\right\|_{J}^{2}\right), \\
& \Theta_{5} \leq \frac{C^{\dagger}}{\alpha_{5}} \frac{\tau^{4}}{h_{x}^{2}}+\alpha_{5} h_{x} \sum_{i}\left(\left\|\xi_{x}^{n+1, C}\left(x_{i}, \cdot\right)\right\|_{J}^{2}+\left\|\xi_{x}^{n+1, D}\left(x_{i+\frac{1}{2}}, \cdot\right)\right\|_{J}^{2}\right),
\end{aligned}
$$

with any $\alpha_{3}, \alpha_{4}, \alpha_{5}>0$. Now we combine the estimates for all $\Theta_{i}, i=1, \ldots, 5$ with (4.40), and obtain

$$
\begin{aligned}
& \frac{1}{2} \sum_{i}\left(\left\|\left.\xi_{x}^{n+1, C}\left(x_{i}, \cdot\right)\right|_{J} ^{2}+\right\| \xi_{x}^{n+1, D}\left(x_{i+\frac{1}{2}}, \cdot\right)\left\|_{J}^{2}-\right\| \xi_{x}^{n, C}\left(x_{i}, \cdot\right)\left\|_{J}^{2}-\right\| \xi_{x}^{n, D}\left(x_{i+\frac{1}{2}}, \cdot\right) \|_{J}^{2}\right) \\
\leq & \theta^{2} \frac{C^{\dagger}}{\alpha_{3}} \frac{\left(h_{x}+h_{y}\right)^{4} h_{y}}{h_{x}^{2}}+\left(\left|u_{x}\right|+\left|u_{y}\right|\right)^{2} \frac{C^{\dagger}}{\alpha_{4}} \frac{\tau^{2}\left(h_{x}+h_{y}\right)^{6}}{h_{x}^{2} h_{y}}+\frac{C^{\dagger}}{\alpha_{5}} \frac{\tau^{4}}{h_{x}^{2}} \\
& +\left(\alpha_{3}+\alpha_{4}+\alpha_{5}\right) h_{x} \sum_{i}\left(\left\|\xi_{x}^{n+1, C}\left(x_{i}, \cdot\right)\right\|_{J}^{2}+\left\|\xi_{x}^{n+1, D}\left(x_{i+\frac{1}{2}}, \cdot\right)\right\|_{J}^{2}\right) \\
& +\left(-\frac{1}{2}+\alpha_{1}+\frac{\alpha_{2}\left(\left|u_{x}\right|+\left|u_{y}\right|\right) \tau}{h_{y}}\right) \sum_{i}\left\|\xi_{x}^{n+1, C}\left(x_{i}, \cdot\right)-\xi_{x}^{n, C}\left(x_{i}, \cdot\right)\right\|_{J}^{2} \\
& +\left(-\frac{1}{2}+\alpha_{1}+\frac{\alpha_{2}\left(\left|u_{x}\right|+\left|u_{y}\right|\right) \tau}{h_{y}}\right) \sum_{i}\left\|\xi_{x}^{n+1, D}\left(x_{i+\frac{1}{2}}, \cdot\right)-\xi_{x}^{n, D}\left(x_{i+\frac{1}{2}}, \cdot\right)\right\|_{J}^{2} \\
& +\left(-\frac{\theta}{2}+\frac{\theta^{2}}{4 \alpha_{1}}+\frac{\left|u_{y}\right| \tau}{\alpha_{2} h_{y}}\right) \sum_{i} \sum_{s=i \pm \frac{1}{2}}\left\|\xi_{x}^{n, D}\left(x_{s}, \cdot\right)-\xi_{x}^{n, C}\left(x_{i}, \cdot\right)\right\|_{J}^{2} \\
& +\frac{\left|u_{x}\right| \tau}{\alpha_{2} h_{x}} \sum_{j} \sum_{s=j \pm \frac{1}{2}}\left\|\xi_{y}^{n, D}\left(\cdot, y_{s}\right)-\xi_{y}^{n, C}\left(\cdot, y_{j}\right)\right\|_{I}^{2} .
\end{aligned}
$$

If formally switching $x$ to $y, i$ to $j, J$ to $I$, we can also get the bound for

$$
\frac{1}{2} \sum_{j}\left(\left\|\xi_{y}^{n+1, C}\left(\cdot, y_{j}\right)\right\|_{I}^{2}+\left\|\xi_{y}^{n+1, D}\left(\cdot, y_{j+\frac{1}{2}}\right)\right\|_{I}^{2}-\left\|\xi_{y}^{n, C}\left(\cdot, y_{j}\right)\right\|_{I}^{2}-\left\|\xi_{y}^{n, D}\left(\cdot, y_{j+\frac{1}{2}}\right)\right\|_{I}^{2}\right)
$$


Combining these bounds with $h_{x}$ as an weight for (4.47) and $h_{y}$ as an weight for the estimate of the $y$ component, taking $\alpha_{3}=\alpha_{4}=\alpha_{5}=\frac{1}{6}$, we get

$$
\begin{aligned}
& \frac{1}{2}\left(\mathcal{E}_{h}\left(\xi^{n+1, C}, \xi^{n+1, D}\right)-\mathcal{E}_{h}\left(\xi^{n, C}, \xi^{n, D}\right)\right) \\
\leq & 6 \Theta+\frac{h_{x}+h_{y}}{2} \mathcal{E}_{h}\left(\xi^{n+1, C}, \xi^{n+1, D}\right) \\
& +h_{x}\left(-\frac{1}{2}+\alpha_{1}+\frac{\alpha_{2}\left(\left|u_{x}\right|+\left|u_{y}\right|\right) \tau}{h_{y}}\right) \sum_{i}\left\{\left\|\xi_{x}^{n+1, C}\left(x_{i}, \cdot\right)-\xi_{x}^{n, C}\left(x_{i}, \cdot\right)\right\|_{J}^{2}+\left\|\xi_{x}^{n+1, D}\left(x_{i+\frac{1}{2}}, \cdot\right)-\xi_{x}^{n, D}\left(x_{i+\frac{1}{2}}, \cdot\right)\right\|_{J}^{2}\right\} \\
& +h_{y}\left(-\frac{1}{2}+\alpha_{1}+\frac{\alpha_{2}\left(\left|u_{x}\right|+\left|u_{y}\right|\right) \tau}{h_{x}}\right) \sum_{j}\left\{\left\|\xi_{y}^{n+1, C}\left(\cdot, y_{j}\right)-\xi_{y}^{n, C}\left(\cdot, x_{i}\right)\right\|_{I}^{2}+\left\|\xi_{y}^{n+1, D}\left(\cdot, y_{j+\frac{1}{2}}\right)-\xi_{y}^{n, D}\left(\cdot, y_{j+\frac{1}{2}}\right)\right\|_{I}^{2}\right\} \\
& +h_{x}\left(-\frac{\theta}{2}+\frac{\theta^{2}}{4 \alpha_{1}}+\frac{\left|u_{y}\right| \tau}{\alpha_{2} h_{y}}+\frac{\left|u_{y}\right| \tau}{\alpha_{2} h_{x}}\right) \sum_{i} \sum_{s=i \pm \frac{1}{2}}\left\|\xi_{x}^{n, D}\left(x_{s}, \cdot\right)-\xi_{x}^{n, C}\left(x_{i}, \cdot\right)\right\|_{J}^{2} \\
& +h_{y}\left(-\frac{\theta}{2}+\frac{\theta^{2}}{4 \alpha_{1}}+\frac{\left|u_{x}\right| \tau}{\alpha_{2} h_{x}}+\frac{\left|u_{x}\right| \tau}{\alpha_{2} h_{y}}\right) \sum_{j} \sum_{s=j \pm \frac{1}{2}}\left\|\xi_{y}^{n, D}\left(\cdot, y_{j-\frac{1}{2}}\right)-\xi_{y}^{n, C}\left(\cdot, y_{j}\right)\right\|_{I}
\end{aligned}
$$

where

$$
\begin{aligned}
\Theta & =C^{\dagger}\left(\theta^{2}\left(h_{x}+h_{y}\right)^{4}\left(\frac{h_{y}}{h_{x}}+\frac{h_{x}}{h_{y}}\right)+\left(\left|u_{x}\right|+\left|u_{y}\right|\right)^{2} \tau^{2}\left(h_{x}+h_{y}\right)^{6} \frac{1}{h_{x} h_{y}}+\tau^{4}\left(\frac{1}{h_{x}}+\frac{1}{h_{y}}\right)\right) \\
& \leq C^{\dagger}\left(\theta^{2} \frac{\left(h_{x}+h_{y}\right)^{6}}{h_{x} h_{y}}+\left(\left|u_{x}\right|+\left|u_{y}\right|\right)^{2} \frac{\tau^{2}\left(h_{x}+h_{y}\right)^{6}}{h_{x} h_{y}}+\frac{\tau^{4}\left(h_{x}+h_{y}\right)}{h_{x} h_{y}}\right) .
\end{aligned}
$$

By requiring all the coefficients of the last four terms of (4.48) to be non-positive. and choosing $\alpha_{1}$ and $\alpha_{2}$ exactly the same way as the ones in Section 4.1.2, we will obtain the same time step condition as for numerical stability in (4.2), $\tau \leq \tau_{\text {stab }}$. Moreover, under this time step condition, we have

$$
\mathcal{E}_{h}\left(\xi^{n+1, C}, \xi^{n+1, D}\right)-\mathcal{E}_{h}\left(\xi^{n, C}, \xi^{n, D}\right) \leq \Theta+\left(h_{x}+h_{y}\right) \mathcal{E}_{h}\left(\xi^{n+1, C}, \xi^{n+1, D}\right) .
$$

\subsubsection{The final step of error estimates}

Define $\Phi_{n}=\left(1-h_{x}-h_{y}\right)^{n} \mathcal{E}_{h}\left(\xi^{n, C}, \xi^{n, D}\right)$, then (4.49) implies $\Phi_{n+1} \leq \Phi_{n}+\Theta\left(1-h_{x}-h_{y}\right)^{n}$. With $\Phi_{0}=0$ and the assumption that $h<\frac{1}{2}$, we have

$$
\Phi_{n} \leq \Theta \sum_{k=0}^{n-1}\left(1-h_{x}-h_{y}\right)^{k} \leq \frac{\Theta}{h_{x}+h_{y}},
$$

and therefore

$$
\begin{aligned}
& \mathcal{E}_{h}\left(\xi^{n, C}, \xi^{n, D}\right)=\left(1-h_{x}-h_{y}\right)^{-n} \frac{\Theta}{h_{x}+h_{y}} \leq \exp \left(n\left(h_{x}+h_{y}\right)\right) \frac{\Theta}{h_{x}+h_{y}} \\
& \leq C^{\dagger} \exp \left(T \frac{h_{x}+h_{y}}{\tau}\right)\left(\theta^{2} \frac{\left(h_{x}+h_{y}\right)^{5}}{h_{x} h_{y}}+\left(\left|u_{x}\right|+\left|u_{y}\right|\right)^{2} \frac{\tau^{2}\left(h_{x}+h_{y}\right)^{5}}{h_{x} h_{y}}+\frac{\tau^{4}}{h_{x} h_{y}}\right) .
\end{aligned}
$$


Now we can apply triangle inequality based on (4.28), the norm equivalency on $\mathcal{M}_{h}^{\star}$ in Lemma 2.1, the approximation property of the projection operators (hence the discrete spaces) in (2.5), and get

$$
\begin{aligned}
& \left\|\mathbf{B}^{n}-\mathbf{B}_{h}^{n, C}\right\|_{\Omega}^{2}+\left\|\mathbf{B}^{n}-\mathbf{B}_{h}^{n, D}\right\|_{\Omega}^{2} \\
& \leq 2\left(\left\|\xi^{n, C}\right\|_{\Omega}^{2}+\left\|\xi^{n, D}\right\|_{\Omega}^{2}+\left\|\eta^{n, C}\right\|_{\Omega}^{2}+\left\|\eta^{n, D}\right\|_{\Omega}^{2}\right) \\
& \leq 2\left(\mathcal{E}_{h}\left(\xi^{n, C}, \xi^{n, D}\right)+\left\|\eta^{n, C}\right\|_{\Omega}^{2}+\left\|\eta^{n, D}\right\|_{\Omega}^{2}\right) \\
& \leq C^{\dagger}\left(\exp \left(T \frac{h_{x}+h_{y}}{\tau}\right)\left\{\theta^{2} \frac{\left(h_{x}+h_{y}\right)^{5}}{h_{x} h_{y}}+\left(\left|u_{x}\right|+\left|u_{y}\right|\right)^{2} \frac{\tau^{2}\left(h_{x}+h_{y}\right)^{5}}{h_{x} h_{y}}+\frac{\tau^{4}}{h_{x} h_{y}}\right\}+h^{2}\right) .
\end{aligned}
$$

This concludes the error estimate in (4.24).

If we further assume that $h_{x}$ and $h_{y}$ satisfy (4.25) during mesh refinement, and denote $\tau=\sigma \tau_{\text {stab }}$ with $\sigma \leq 1$, the error estimate can be expressed in a simpler form in (4.26).

\section{NUMERICAL EXPERIMENTS}

In this section, we demonstrate the performance of the scheme through some numerical experiments and verify some of the theoretical results. In particular, we will examine the sharpness of the CFL condition (3.6) in Theorem 3.1, and illustrate the convergence order of the method when the velocity field $\mathbf{u}$ is constant and when it is spatially dependent.

We start with an example defined on the computational domain $\Omega=[0,1] \times[0,1]$ with a constant velocity field $\mathbf{u}=\left[u_{x}, u_{y}\right]^{\top}=[1,1]^{\top}$. The initial condition is

$$
B_{x}(x, y, 0)=-\sin (2 \pi y), \quad B_{y}(x, y, 0)=\sin (2 \pi x),
$$

and the exact solution is

$$
B_{x}(x, y, t)=-\sin (2 \pi(y-t)), \quad B_{y}(x, y, t)=\sin (2 \pi(x-t))
$$

at any time $t$. We use this example to demonstrate numerically how sharp the CFL condition in (3.6) is. To this end, we take the parameter $\theta$ to be $0.1,0.2, \ldots, 1.0$, respectively, and implement the scheme on a uniform mesh with $h_{x}=h_{y}=h=\frac{1}{40}$ and periodic boundary conditions. We then report in Table 1 the largest value of $\sqrt{\left(\frac{\tau u_{x}}{h_{x}}\right)^{2}+\left(\frac{\tau u_{y}}{h_{y}}\right)^{2}}$, denoted as $C_{h, \theta}$, with which the scheme is stable in long term simulation. The number $C_{h, \theta}$ is the largest in the sense that the scheme blows up if the corresponding $\frac{\tau}{h}$ increases to $\frac{\tau}{h}+\varepsilon$, with $\varepsilon=0.01$ in our experiment. In Table 1, we also include $\frac{\sqrt{\theta}}{2}$, a sufficient upper bound of $\sqrt{\left(\frac{\tau u_{x}}{h_{x}}\right)^{2}+\left(\frac{\tau u_{y}}{h_{y}}\right)^{2}}$ for numerical stability based on Fourier analysis in Theorem 3.1. One can see $C_{h, \theta}>\frac{\sqrt{\theta}}{2}$, and this is consistent with that the CFL condition in (3.6) is only sufficient. On the other hand, our analytical bound $\frac{\sqrt{\theta}}{2}$ is a fairly good approximation to $C_{h, \theta}$, and hence the result in (3.6) provides a practically useful CFL condition.

Another way to examine the sharpness of the CFL condition in (3.6) is to directly compare the ranges of $\lambda_{x}=\frac{\tau u_{x}}{h_{x}}$ and $\lambda_{y}=\frac{\tau u_{y}}{h_{y}}$ allowed by this condition and by the sufficient and necessary condition in (3.5). This is demonstrated by the plots in Figure 2 with $\theta=1, \frac{3}{4}, \frac{1}{2}$ and $\frac{3}{8}$. In each plot, the blue dotted region consists of the values of $\left(2 \lambda_{x}, 2 \lambda_{y}\right)$ permitted by (3.5), and the red solid curve corresponds to the circle $\left(2 \lambda_{x}\right)^{2}+\left(2 \lambda_{y}\right)^{2}=\theta$ arising from the condition (3.6). With symmetry, the plots are only shown in the first quadrant. One can again see that the result in (3.6) provides a good CFL condition to guide how the time step is chosen for stable simulations.

With the same exact solution and the constant velocity field used above, we next compute the $L^{2}$ errors and convergence orders of the scheme with $\theta=1$ when it is implemented on a sequence of refined meshes with $h=\frac{1}{N}, N=20,40,80,160$. The time step $\tau$ is the largest allowed by the CFL condition (3.6). In Table 2, three 
TABle 1. Study of the sharpness of the CFL condition in (3.6).

\begin{tabular}{ccc}
\hline$\theta$ & $C_{h, \theta}$ & $\frac{\sqrt{\theta}}{2}$ \\
\hline 1.0 & 0.5091 & 0.5000 \\
0.9 & 0.4808 & 0.4743 \\
0.8 & 0.4525 & 0.4472 \\
0.7 & 0.4243 & 0.4183 \\
0.6 & 0.3960 & 0.3873 \\
0.5 & 0.3677 & 0.3536 \\
0.4 & 0.3253 & 0.3162 \\
0.3 & 0.2828 & 0.2739 \\
0.2 & 0.2263 & 0.2236 \\
0.1 & 0.1697 & 0.1581 \\
\hline
\end{tabular}

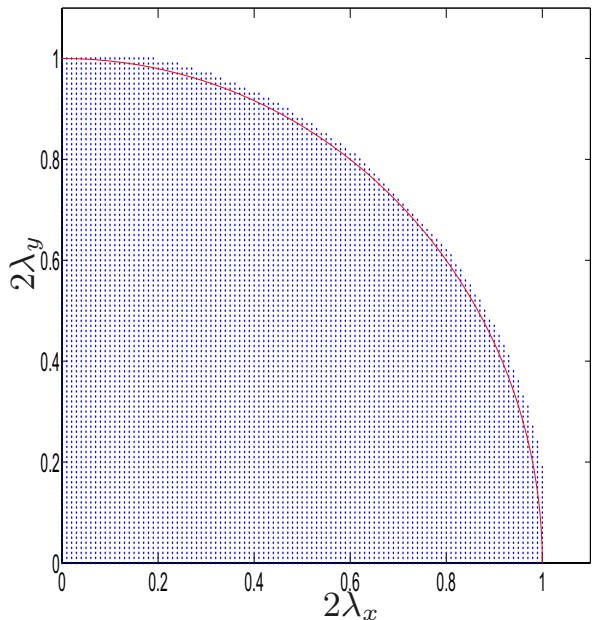

(a) $\theta=1$

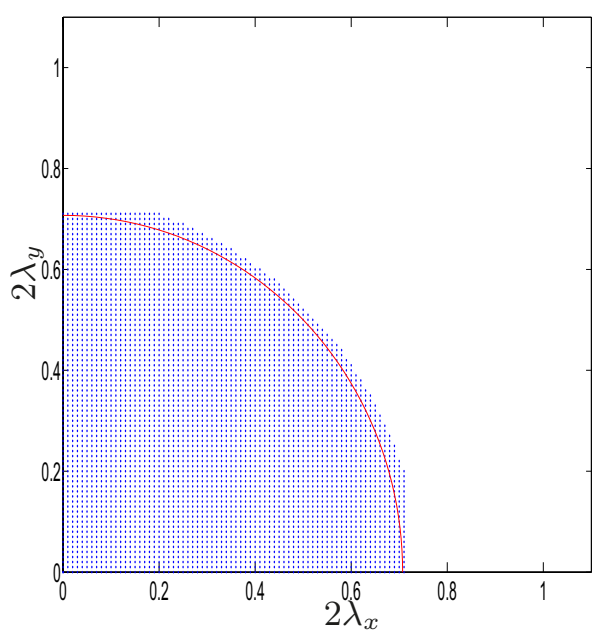

(c) $\theta=1 / 2$

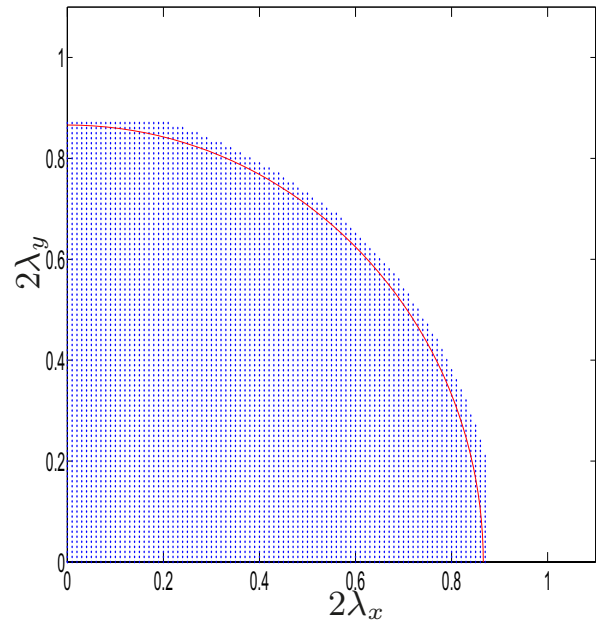

(b) $\theta=3 / 4$

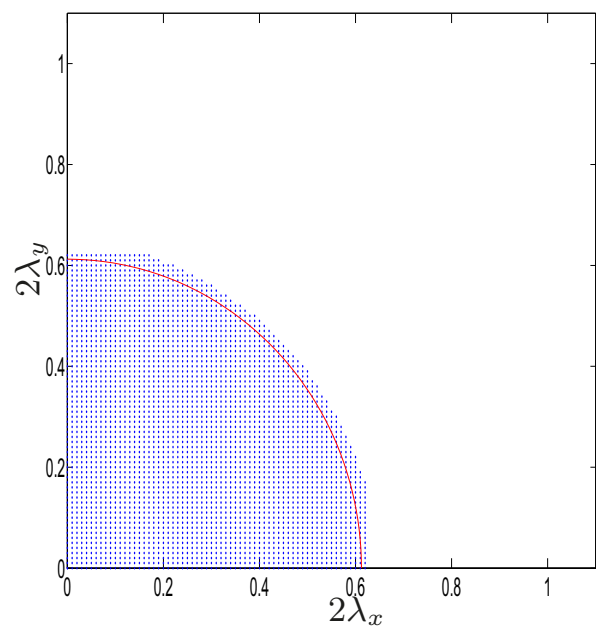

(d) $\theta=3 / 8$

Figure 2. Comparison of the CFL conditions in (3.5) and (3.6). 
TABLE 2. $L^{2}$ errors and convergence orders of the scheme with $\theta=1$ on a uniform mesh with $h=\frac{1}{N}$. The velocity field is constant.

\begin{tabular}{ccccc}
\hline$N$ & $e r r C$ & $e r r D$ & $e r r$ & order \\
\hline 20 & $3.00 \mathrm{E}-1$ & $3.00 \mathrm{E}-1$ & $4.19 \mathrm{E}-1$ & - \\
40 & $1.63 \mathrm{E}-1$ & $1.63 \mathrm{E}-1$ & $2.28 \mathrm{E}-1$ & 0.88 \\
80 & $8.41 \mathrm{E}-2$ & $8.41 \mathrm{E}-2$ & $1.19 \mathrm{E}-1$ & 0.95 \\
160 & $4.28 \mathrm{E}-2$ & $4.28 \mathrm{E}-2$ & $6.05 \mathrm{E}-2$ & 0.98 \\
\hline
\end{tabular}

TABLE 3. $L^{2}$ errors and convergence orders of the scheme with $\theta=1$ on a uniform mesh with $h=\frac{1}{N}$. The velocity field is spatially dependent.

\begin{tabular}{ccccc}
\hline$N$ & $e r r C$ & $e r r D$ & $e r r$ & order \\
\hline 20 & $5.43 \mathrm{E}-1$ & $5.43 \mathrm{E}-1$ & $7.69 \mathrm{E}-1$ & - \\
40 & $3.61 \mathrm{E}-1$ & $3.61 \mathrm{E}-1$ & $5.10 \mathrm{E}-1$ & 0.59 \\
80 & $2.19 \mathrm{E}-1$ & $2.19 \mathrm{E}-1$ & $3.10 \mathrm{E}-1$ & 0.72 \\
160 & $1.23 \mathrm{E}-1$ & $1.23 \mathrm{E}-1$ & $1.73 \mathrm{E}-1$ & 0.84 \\
320 & $6.52 \mathrm{E}-2$ & $6.52 \mathrm{E}-2$ & $9.23 \mathrm{E}-2$ & 0.91 \\
640 & $3.38 \mathrm{E}-2$ & $3.38 \mathrm{E}-2$ & $4.77 \mathrm{E}-2$ & 0.95 \\
\hline
\end{tabular}

types of errors at $T=1$ are presented, and they are $\operatorname{err} C=\left\|\mathbf{B}^{n}-\mathbf{B}_{h}^{n, C}\right\|_{0, \Omega}, \operatorname{err} D=\left\|\mathbf{B}^{n}-\mathbf{B}_{h}^{n, D}\right\|_{0, \Omega}$, and $e r r=\sqrt{(e r r C)^{2}+(e r r D)^{2}}$. The convergence orders in the last column are computed based on the total error, err. Our results confirm the first order accuracy as proved in Theorem 4.6 for the proposed method.

Finally, we illustrate the accuracy of the proposed scheme when the velocity field is spatially dependent. Here we make use of the following time reversible property of the magnetic induction equations with periodic boundary conditions: let $\mathbf{B}(x, y, T)$ denote the exact solution of the equations with the velocity field $\mathbf{u}=\mathbf{v}$ at time $T$. If we start with $\mathbf{B}(x, y, T)$ as the initial data, and consider the magnetic induction equations with a different velocity field $\mathbf{u}=-\mathbf{v}$, then $\mathbf{B}(x, y, 0)$ will give the exact solution at time $T$ for this new problem. In our simulation, the scheme is run first from $t=0$ to $t=0.5$ with the velocity field $\mathbf{u}=[-\sin (2 \pi y), 1]^{\top}$ and the initial condition (5.1). We then use the computed solution at $T=0.5$ as the initial condition, take the negative velocity field, i.e. $\mathbf{u}=[\sin (2 \pi y),-1]^{\top}$, and run the scheme for another time interval from $t=0$ to $t=0.5$. We compare the final computed solution with the initial data (5.1). The $L^{2}$ errors and convergence orders are presented in Table 3 . The results again confirm the first order accuracy of the proposed scheme.

\section{Concluding REMarks}

As an effort to gain better theoretical understanding of the constrained transport type divergence-free schemes, we analyze in this work a first order exactly divergence-free method on overlapping Cartesian meshes for the magnetic induction equations, a linear problem extracted from ideal MHD equations. Numerical stability is established through both Fourier and energy methods when the meshes are uniform and when the velocity field in the equations is constant. A priori error estimate is also obtained in the $L^{2}$ norm for sufficiently smooth solutions.

Though not being the focus of this paper, stability and error estimates based on energy methods can be extended to more general cases, including non-uniform Cartesian meshes and variable velocity fields. The generalization to the non-uniform mesh case is straightforward, as long as some reasonable assumption is made on how non-uniform the meshes are. In the case of the variable velocity fields, a more relevant case to the MHD simulations, the exact solution satisfies

$$
\frac{\mathrm{d}}{\mathrm{d} t} \int_{\Omega}|\mathbf{B}|^{2} \mathrm{~d} x \mathrm{~d} y \leq C\left(|\mathbf{u}(\cdot, \cdot, t)|_{W^{1, \infty}(\Omega)}\right) \int_{\Omega}|\mathbf{B}|^{2} \mathrm{~d} x \mathrm{~d} y,
$$


where $|\cdot|_{W^{1, \infty}(\Omega)}$ is the semi $W^{1, \infty}$-norm. In Appendix, numerical stability analysis is given based on energy approach when the velocity field is spatially dependent on uniform meshes.

The mathematical understanding gained for the first order divergence-free scheme examined here, together with the analysis framework based on energy approach, provides us a starting point to further study some other divergence-free schemes, such as those with higher order accuracy, defined on one mesh, and in higher dimensions, or even with more general boundary conditions. New technical challenges are expected and would need to be addressed.

\section{Appendix A. Numerical Stability by EnERgy Methods: SPATIALly DEPEndent VELOCITY FIELD}

In this appendix, we will present the stability analysis by energy methods when the velocity field $\mathbf{u}$ is spatially dependent.

Theorem A.1. Given any $\theta \in(0,1)$, the numerical solutions of the proposed method in $(2.6)-(2.8)$ with a variable velocity field $\mathbf{u}$ satisfy

$$
\mathcal{E}_{h}\left(\mathbf{B}_{h}^{n, C}, \mathbf{B}_{h}^{n, D}\right) \leq e^{\left(\mathcal{C}_{\theta}|\mathbf{u}|_{W^{1, \infty}(\Omega)} T\right)} \mathcal{E}_{h}\left(\mathbf{B}_{h}^{0, C}, \mathbf{B}_{h}^{0, D}\right), \quad \forall n: n \tau \leq T,
$$

under the following CFL condition on the time step $\tau$

$$
\tau<\tau_{s t a b}
$$

Here the constant $\mathcal{C}_{\theta}$ depends on $\frac{h_{x}}{h_{y}}, \frac{h_{y}}{h_{x}}$ and $\theta$, and $\tau_{\text {stab }}$ is defined in (4.2).

Proof. Following the proof in Section 4.1.2, we only need to re-estimate $\Lambda_{2}$ and $\Lambda_{4}$. Without loss of generality, it is assumed $\tau \leq 1$.

For $\Lambda_{2}$, we have $\Lambda_{2}=\Lambda_{21}+\Lambda_{22}$, where

$$
\begin{aligned}
\Lambda_{21}= & \tau \sum_{i, j}\left(-u_{y, i, j+1} b_{x, i, j+1}^{n, D}+u_{y, i, j} b_{x, i, j}^{n, D}\right) b_{x, i, j+\frac{1}{2}}^{n, C} \\
& +\tau \sum_{i, j}\left(-u_{y, i+\frac{1}{2}, j+\frac{1}{2}} b_{x, i+\frac{1}{2}, j+\frac{1}{2}}^{n, C}+u_{y, i+\frac{1}{2}, j-\frac{1}{2}} b_{x, i+\frac{1}{2}, j-\frac{1}{2}}^{n, C}\right) b_{x, i+\frac{1}{2}, j}^{n, D} \\
= & \frac{\tau}{2} \sum_{i, j}\left(-u_{y, i+\frac{1}{2}, j+\frac{1}{2}}+u_{y, i, j}\right) b_{x, i, j+\frac{1}{2}}^{n, C} b_{x, i+\frac{1}{2}, j}^{n, D} \\
& +\frac{\tau}{2} \sum_{i, j}\left(-u_{y, i-\frac{1}{2}, j+\frac{1}{2}}+u_{y, i, j}\right) b_{x, i, j+\frac{1}{2}}^{n, C} b_{x, i-\frac{1}{2}, j}^{n, D} \\
& +\frac{\tau}{2} \sum_{i, j}\left(-u_{y, i, j+1}+u_{y, i+\frac{1}{2}, j+\frac{1}{2}}\right) b_{x, i, j+\frac{1}{2}}^{n, C} b_{x, i+\frac{1}{2}, j+1}^{n, D} \\
& +\frac{\tau}{2} \sum_{i, j}\left(-u_{y, i, j+1}+u_{y, i-\frac{1}{2}, j+\frac{1}{2}}\right) b_{x, i, j+\frac{1}{2}}^{n, C} b_{x, i-\frac{1}{2}, j+1}^{n, D} \\
\leq & \mathcal{C} \tau\left|u_{y}\right|_{W^{1, \infty}(\Omega)} \sum_{i}\left(\left\|b_{x, i}^{n, C}\right\|_{J}^{2}+\left\|b_{x, i+\frac{1}{2}}^{n, D}\right\|_{J}^{2}\right)
\end{aligned}
$$


and

$$
\begin{aligned}
\Lambda_{22}= & \tau \sum_{i, j}\left(u_{x, i, j+1} b_{y, i, j+1}^{n, D}-u_{x, i, j} b_{y, i, j}^{n, D}\right) b_{x, i, j+\frac{1}{2}}^{n, C} \\
& +\tau \sum_{i, j}\left(u_{x, i+\frac{1}{2}, j+\frac{1}{2}} b_{y, i+\frac{1}{2}, j+\frac{1}{2}}^{n, C}-u_{x, i+\frac{1}{2}, j-\frac{1}{2}} b_{y, i+\frac{1}{2}, j-\frac{1}{2}}^{n, C}\right) b_{x, i+\frac{1}{2}, j}^{n, D} \\
= & \frac{\tau}{2} \sum_{i, j}\left(u_{x, i, j+1}-u_{x, i, j}\right)\left(b_{y, i, j+\frac{1}{2}}^{n, D}+b_{y, i, j+\frac{3}{2}}^{n, D}\right) b_{x, i, j+\frac{1}{2}}^{n, C} \\
& +\frac{\tau}{2} \sum_{i, j}\left(u_{x, i+\frac{1}{2}, j+\frac{1}{2}}-u_{x, i, j}\right)\left(b_{y, i+\frac{1}{2}, j}^{n, C}+b_{y, i+\frac{1}{2}, j+1}^{n, C}\right) b_{x, i+\frac{1}{2}, j}^{n, D} \\
& +\frac{\tau}{2} \sum_{i, j}\left(u_{x, i, j}-u_{x, i+\frac{1}{2}, j-\frac{1}{2}}\right)\left(b_{y, i+\frac{1}{2}, j-1}^{n, C}+b_{y, i+\frac{1}{2}, j}^{n, C}\right) b_{x, i+\frac{1}{2}, j}^{n, D} \\
& -\frac{\tau}{2} \frac{h_{y}}{h_{x}} \sum_{i, j}\left(u_{x, i-1, j}-u_{x, i, j}\right) b_{x, i, j+\frac{1}{2}}^{n, C} b_{x, i-\frac{1}{2}, j}^{n, D} \\
& -\frac{\tau}{2} \frac{h_{y}}{h_{x}} \sum_{i, j}\left(u_{x, i, j}-u_{x, i, j+1}\right) b_{x, i, j+\frac{1}{2}}^{n, C} b_{x, i+\frac{1}{2}, j+1}^{n, D} \\
& -\frac{\tau}{2} \frac{h_{y}}{h_{x}} \sum_{i, j}\left(-u_{x, i, j}+u_{x, i-1, j+1}\right) b_{x, i, j+\frac{1}{2}}^{n, C} b_{x, i-\frac{1}{2}, j+1}^{n, D} \\
\leq & \mathcal{C} \tau\left|u_{x}\right|_{W^{1, \infty}(\Omega)}\left(\sum_{i}\left(\left\|b_{x, i}^{n, C}\right\|_{J}^{2}+\left\|b_{x, i+\frac{1}{2}}^{n, D}\right\|_{J}^{2}\right)+\sum_{j}\left(\left\|b_{y, j}^{n, C}\right\|_{I}^{2}+\left\|b_{y, j+\frac{1}{2}}^{n, D}\right\|_{I}^{2}\right)\right) .
\end{aligned}
$$

Note that the numerical solution being divergence-free has been used to estimate $\Lambda_{22}$. Here and below, the constant $\mathcal{C}$ depends on $\frac{h_{x}}{h_{y}}$ and $\frac{h_{y}}{h_{x}}$, and it may take different values at different occurrences. We use shorthand notation such as $u_{y, i, j}$ which is to denote $u_{y}\left(x_{i}, y_{j}\right)$.

We next consider $\Lambda_{4}=\Lambda_{41}+\Lambda_{42}$, where

$$
\begin{aligned}
& \Lambda_{41}=\tau \sum_{i, j}\left(-E_{z, h}^{n, D}\left(x_{i}, y_{j+1}\right)+E_{z, h}^{n, D}\left(x_{i}, y_{j}\right)\right)\left(b_{x, i, j+\frac{1}{2}}^{n+1, C}-b_{x, i, j+\frac{1}{2}}^{n, C}\right), \\
& \Lambda_{42}=\tau \sum_{i, j}\left(-E_{z, h}^{n, C}\left(x_{i+\frac{1}{2}}, y_{j+\frac{1}{2}}\right)+E_{z, h}^{n, C}\left(x_{i+\frac{1}{2}}, y_{j-\frac{1}{2}}\right)\right)\left(b_{x_{i+\frac{1}{2}, j}^{n+1, D}}^{n+b_{x_{i+\frac{1}{2}}, j}^{n, D}}\right) .
\end{aligned}
$$

With similarity, we only estimate $\Lambda_{41}$. It is easy to see that $\Lambda_{41}$ can be written as the sum of

$$
\begin{aligned}
& \frac{\tau}{2} \sum_{i, j}\left(-u_{y, i, j+1}+u_{y, i, j}\right)\left(b_{x, i-\frac{1}{2}, j+1}^{n, D}+b_{x, i+\frac{1}{2}, j+1}^{n, D}\right)\left(b_{x, i, j+\frac{1}{2}}^{n+1, C}-b_{x, i, j+\frac{1}{2}}^{n, C}\right) \\
& \leq \tau\left\|\frac{\partial u_{y}}{\partial y}\right\|_{L^{\infty}(\Omega)}\left(\sum_{i}\left\|b_{x, i+\frac{1}{2}}^{n, D}\right\|_{J}^{2}\right)^{1 / 2}\left(\sum_{i}\left\|B_{x, h}^{n+1, C}\left(x_{i}, \cdot\right)-B_{x, h}^{n, C}\left(x_{i}, \cdot\right)\right\|_{J}^{2}\right)^{1 / 2} \\
& \leq \frac{1}{\epsilon} \tau\left|u_{y}\right|_{W^{1, \infty}(\Omega)} \sum_{i}\left\|b_{x, i+\frac{1}{2}}^{n, D}\right\|_{J}^{2}+\epsilon\left|u_{y}\right|_{W^{1, \infty}(\Omega)} \sum_{i}\left\|B_{x, h}^{n+1, C}\left(x_{i}, \cdot\right)-B_{x, h}^{n, C}\left(x_{i}, \cdot\right)\right\|_{J}^{2}
\end{aligned}
$$

(with arbitrary constant $\epsilon>0$ ) and

$$
\frac{\tau}{2} \sum_{i, j} u_{x, i, j}\left(-b_{x, i-\frac{1}{2}, j+1}^{n, D}-b_{x, i+\frac{1}{2}, j+1}^{n, D}+b_{x, i-\frac{1}{2}, j}^{n, D}+b_{x, i+\frac{1}{2}, j}^{n, D}\right)\left(b_{x, i, j+\frac{1}{2}}^{n+1, C}-b_{x, i, j+\frac{1}{2}}^{n, C}\right) .
$$


One can see that the term (A.6) can be estimated just as in the constant velocity case, see (4.19) with $u_{x}$ and $u_{y}$ replaced by their $L^{\infty}$ norms.

Combining the new estimates of $\Lambda_{2}$ and $\Lambda_{4}$, and using the "symmetric" structure in the different components of the computed solution, we eventually have

$$
\frac{1}{2}\left(\mathcal{E}_{h}\left(\mathbf{B}_{h}^{n+1, C}, \mathbf{B}_{h}^{n+1, D}\right)-\mathcal{E}_{h}\left(\mathbf{B}_{h}^{n, C}, \mathbf{B}_{h}^{n, D}\right)\right) \leq \aleph_{\epsilon}+\mathcal{C}_{\epsilon} \tau|\mathbf{u}|_{W^{1, \infty}(\Omega)} \mathcal{E}_{h}\left(\mathbf{B}_{h}^{n, C}, \mathbf{B}_{h}^{n, D}\right) .
$$

Here $\mathcal{C}_{\epsilon}$ depends on $\frac{h_{x}}{h_{y}}, \frac{h_{y}}{h_{x}}$ and $\epsilon$. The term $\aleph_{\epsilon}$ is almost identical to the right hand side of (4.21), except that $\left|u_{x}\right|$ and $\left|u_{y}\right|$ are replaced by their $L^{\infty}$ norms, and all four $\left(-\frac{1}{2}\right)$ s are replaced by $-\frac{1}{2}+\epsilon|\mathbf{u}|_{W^{1, \infty}(\Omega)}$. Similar as in Section 4.1.2, for any given $\theta \in(0,1)$, one can choose $\epsilon$ such that $\aleph_{\epsilon} \leq 0$ as long as $\tau$ satisfies (A.2). The corresponding $\mathcal{C}_{\epsilon}$ is now denoted as $\mathcal{C}_{\theta}$.

Under this time step condition, we have for any $n: n \tau \leq T$,

$$
\mathcal{E}_{h}\left(\mathbf{B}_{h}^{n, C}, \mathbf{B}_{h}^{n, D}\right) \leq\left(1+\mathcal{C}_{\theta}|\mathbf{u}|_{W^{1, \infty}(\Omega)} \tau\right) \mathcal{E}_{h}\left(\mathbf{B}_{h}^{n-1, C}, \mathbf{B}_{h}^{n-1, D}\right) \leq \mathrm{e}^{\left(\mathcal{C}_{\theta}|\mathbf{u}|_{W^{1, \infty}(\Omega)} T\right)} \mathcal{E}_{h}\left(\mathbf{B}_{h}^{0, C}, \mathbf{B}_{h}^{0, D}\right) .
$$

\section{REFERENCES}

[1] D.S. Balsara, Divergence-free reconstruction of magnetic fields and WENO schemes for magne tohydrodynamics. J. Comput. Phys. 228 (2009) 5040-5056.

[2] D.S. Balsara and D.S. Spicer, A staggered mesh algorithm using high order Godunov fluxes to ensure solenoidal magnetic fields in magnetohydrodynamic simulations. J. Comput. Phys. 149 (1999) 270-292.

[3] T. Barth, On the role of involutions in the discontinuous Galerkin discretization of Maxwell and magnetohydrodynamic systems, The IMA Volumes in Mathematics and its Applications, IMA volume on Compatible Spatial Discretizations, edited by Arnold, Bochev and Shashkov 142 (2005) 69-88.

[4] N. Besse and D. Kröner, Convergence of the locally divergence free discontinuous Galerkin methods for induction equations for the 2D-MHD system. ESAIM: M2AN 39 (2005) 1117-1202.

[5] J.U. Brackbill and D.C. Barnes, The effect of nonzero $\nabla \cdot \mathbf{B}$ on the numerical solution of the magnetohydrodynamic equations. J. Comput. Phys. 35 (1980) 426-430.

[6] F. Brezzi, J. Douglas, Jr. and L.D. Marini, Two Families of Mixed Finite Elements for Second Order Elliptic Problems. Numer. Math. 47 (1985) 217-235.

[7] B. Cockburn, An introduction to the discontinuous Galerkin method for convection-dominated problems. Advanced Numerical Approximation of Nonlinear Hyperbolic Equations. Vol. 169 of Lect. Notes Math. (1998) 151-268.

[8] C.R. Evans and J.F. Hawley, Simulation of magnetohydrodynamic flows: a constrained transport method. Astrophys. J. 322 (1988) 659-677.

[9] F.G. Fuchs, K.H. Karlsen, S. Mishra and N.H. Risebro, Stable upwind schemes for the magnetic induction equation. ESAIM: M2AN 43 (2009) 825-852.

[10] T.A. Gardiner and J.M. Stone, An unsplit Godunov method for ideal MHD via constrained transport. J. Comput. Phys. 205 (2005) 509-539.

[11] S.K. Godunov, Symmetric form of the equations of magnetohydrodynamics. Numer. Methods Mech. Continuum Medium 1 (1972) 26-34.

[12] S. Li, High order central scheme on overlapping cells for magnetohydrodynamic flows with and without constrained transport method. J. Comput. Phys. 227 (2008) 7368-7393.

[13] F. Li and L. Xu, Arbitrary order exactly divergence-free central discontinuous Galerkin methods for ideal MHD equations. J. Comput. Phys. 231 (2012) 2655-2675.

[14] F. Li, L. Xu and S. Yakovlev, Central discontinuous Galerkin methods for ideal MHD equations with the exactly divergence-free magnetic field. J. Comput. Phys. 230 (2011) 4828-4847.

[15] Y. Liu, C.-W. Shu, E. Tadmor and M. Zhang, Central discontinuous Galerkin methods on overlapping cells with a nonoscillatory hierarchical reconstruction. SIAM J. Numer. Anal. 45 (2007) 2442-2467.

[16] Y. Liu, C.-W. Shu, E. Tadmor and M. Zhang, $L^{2}$ stability analysis of the central discontinuous Galerkin method and a comparison between the central and regular discontinuous Galerkin methods. ESAIM: M2AN 42 (2008) 593-607.

[17] S. Mishra and M. Svärd, On stability of numerical schemes via frozen coefficients and the magnetic induction equations. BIT Num. Math. 50 (2010) 85-108.

[18] K.W. Morton and R.L. Roe, Vorticity-preserving Lax-Wendroff-type schemes for the system wave equation. SIAM J. Sci. Comput. 23 (2001) 170-192.

[19] P.A. Raviart and J.M. Thomas, A mixed finite element method for 2nd order elliptic problems, Mathematical Aspects of Finite Element Methods. Springer (1997) 292-315. 
[20] J.A. Rossmanith, An unstaggered, high-resolution constrained transport method for magnetohydrodynamic flows. SIAM J. Sci. Comput. 28 (2006) 1766-1797.

[21] J.A. Rossmanith, High-order discontinuous Galerkin finite element methods with globally divergence-free constrained transport for ideal MHD. Preprint (2013).

[22] G. Tóth, The $\nabla \cdot \mathbf{B}=0$ constraint in shock-capturing magnetohydrodynamics codes. J. Comput. Phys. 161 (2000) 605-652.

[23] Q. Zhang and C-W Shu, Error estimates to smooth solutions of Runge-Kutta discontinuous Galerkin methods for scalar conservation laws. SIAM J. Numer. Anal. 42 (2004) 641-666.

[24] Q. Zhang and C-W Shu, Stability analysis and a priori error estimates to the third order explicit Runge-Kutta discontinuous Galerkin method for scalar conservation laws, SIAM J. Numerical Anal. 48 (2010) 1038-1063. 